BULLETIN (New Series) OF THE

AMERICAN MATHEMATICAL SOCIETY

Volume 46, Number 2, April 2009, Pages 221-254

S 0273-0979(09)01243-9

Article electronically published on January 15, 2009

\title{
REMARKS ON CHERN-SIMONS THEORY
}

\author{
DANIEL S. FREED
}

Dedicated to MSRI on its $25^{\text {th }}$ anniversary

\begin{abstract}
The classical Chern-Simons invariant is the basis for a 3-dimensional topological quantum field theory. We describe some of the mathematical structure which has been built around this and other topological field theories. We include, in the introduction and the last section, some general discussion about the current interaction between geometry and quantum theories of fields and gravity.
\end{abstract}

A beautiful line of development in Riemannian geometry is the relationship between curvature and topology. In one of his first major works, written in 1946, Chern $\mathrm{Ch}$ proves a generalized Gauss-Bonnet theorem by producing what we now call a transgressing form on the unit sphere bundle of the manifold. Twenty-five years later, together with Simons, he took up transgression [CSi] in the context of the theory of connections on arbitrary principal bundles. The Chern-Simons invariants of a connection are secondary geometric invariants; in between these works Chern and Weil developed the theory of primary topological invariants of connections. Both invariants are local in the sense that they are computed by integrals of differential forms. The relationship between them is that the differential of the Chern-Simons form is the Chern-Weil form. The integral of the Chern-Weil form over a closed manifold is independent of the connection, so is a topological invariant. In the late 1980s Edward Witten W1] proposed a new topological invariant of 3-manifolds from these same ingredients. He achieves topological invariance via a technique unavailable to geometers: Witten integrates the exponentiated ChernSimons invariant over the infinite-dimensional space of all connections. Because the connection is integrated out, the result depends only on the underlying manifold. This, then, is the quantum Chern-Simons invariant.

There are rich stories to tell about both the classical and quantum Chern-Simons invariants in geometry, topology, and physics. The classical Chern-Simons invariant is an obstruction to conformal immersions of 3-manifolds into Euclidean space, is closely related to the Atiyah-Patodi-Singer invariant, and was refined in the Cheeger-Simons theory of differential characters. It appeared in physics before Witten's work, for example in the theory of anomalies. The quantum Chern-Simons invariant is closely related to the Jones invariants Jo1 of links, which have had many applications in knot theory. But here we do not attempt a review of all work

Received by the editors August 8, 2008.

2000 Mathematics Subject Classification. Primary 81T45, 53C05, 55N15, 18F99.

The author is supported by NSF grant DMS-0603964.

Based on a talk given in the Simons Auditorium in Chern Hall at the Mathematical Sciences Research Institute on the occasion of its $25^{\text {th }}$ Anniversary.

(C)2009 American Mathematical Society Reverts to public domain 28 years from publication 
on Chern-Simons invariants. Our interest is the structure behind the quantum invariants as they relate to structure in physics. Indeed, Witten's achievement was to fit the Jones invariants into a larger structure, that of a 3-dimensional quantum field theory.

The beginning of the twentieth century saw two revolutionary developments in physics: relativity and quantum mechanics. The mathematical basis for general relativity - differential geometry - was already well developed when Einstein came along, and in turn his gravitational equations, which focus attention on Ricci curvature, spurred many developments in Riemannian geometry. Quantum mechanics, on the other hand, quickly inspired the development of operator theory and parts of representation theory. These foundations for quantum mechanics later influenced many other parts of mathematics, including diverse areas such as partial differential equations and number theory $\mathrm{Ma}$. Quantum field theory in its first incarnation, quantum electrodynamics, combines quantum mechanics and Maxwell's classical theory of the electromagnetic field. It has provided some of the most precise computational agreement between theory and experiment, and quantum field theory is the setting for the standard model of particle physics. Although there has been much mathematical work on quantum field theory, its foundations are not at all settled. The interaction with mathematics has greatly broadened over the past 25 years: now quantum field theory - and also string theory - enjoys a deep interaction with many branches of mathematics, suggesting novel results, surprising connections, and new lines of research. One important impetus for this development was the advent of new examples of quantum field theories closely connected with geometry and topology. Quantum Chern-Simons theory is one of the first examples, and it is a purely topological theory at both the classical and quantum levels. We use it here as a focal point to discuss topological quantum field theories (TQFT) in general. From it we have gained insight into the formal structure of all quantum field theories, not just topological ones, and it is that general structure which we accentuate. Also, Chern-Simons theory provides a window into the entire interchange between mathematics and these parts of physics. We return to this line of thought in the second half of 8 .

Witten's main tool is the path integral, an integral on a function space. (In Feynman's approach to quantum mechanics [Fe] it is truly an integral over a space of paths; in general field theories it is still usually referred to as the "path integral", though "functional integral" is better.) Regrettably, the measures needed for such integrals have only been constructed in special theories, and these do not cover the example of Chern-Simons theory. In $\$ 2$ we extract some important formal properties of path integrals in general. We focus on locality, which is manifested in gluing laws. This formal structure is nicely expressed in familiar mathematical terms, as a linearization of correspondence diagrams. Here the correspondence diagrams are built from the semiclassical fields; the linearization is the quantum theory. Following Segal Se2 and Atiyah [A2, we abstract axioms for a TQFT, which we give in $\$ 3$. The axioms do not encode the passage from fields to the quantum theory, but only the properties of the quantum theory itself. In the end these axioms are quite analogous to those of homology theory, but with important differences: (i) a TQFT is defined on manifolds of a particular dimension, whereas homology theory is defined on arbitrary topological spaces; and (ii) a TQFT is multiplicative (say on disjoint unions) whereas homology theory is additive. The 
axioms are most neatly stated in categorical language, which, given the analogy with homology theory, is not a surprise.

While the definition of a TQFT is simple, examples are not so easy to come by. We distinguish between generators-and-relations constructions and a priori constructions. The former are most prevalent in the mathematics literature, and we present some theorems which classify TQFTs in these terms. (See Theorem 3.8 and Theorem 4.1 for example.) Most rigorous constructions of the 3-dimensional Chern-Simons theory are of this generators-and-relations type. The path integral is an a priori construction - the axioms of a TQFT follow directly and geometricallybut in most cases it is not rigorous; a notable exception is gauge theory with finite gauge group where the path integral reduces to a finite sum. The 2-dimensional reduction of Chern-Simons theory for any gauge group has an a priori construction. In fact, that reduced theory exists over the integers, whereas the 3-dimensional Chern-Simons theory is defined over the complex numbers. It may be that TQFTs which are dimensional reductions always exhibit integrality - and even a connection to $K$-theory - as we speculate in $\$ 5$.

Another theme here is the extension of a TQFT to higher codimension. Usually in a quantum field theory there are two dimensions in play: the dimension $n$ of spacetime and the dimension $n-1$ of space. For sure locality in space appears in the physics literature - think of the Hilbert space of a lattice model constructed as a tensor product of local Hilbert spaces, or of local algebras of observables in some axiomatic treatments of quantum field theory - though that aspect is not always emphasized. One of the very fruitful ideas in topological theories is to consider gluing laws along corners. In the best case one goes all the way down to points. So, for example, the usual 3-dimensional Chern-Simons theory may be called a "2-3 theory" as it involves 2- and 3-dimensional manifolds. The extension down to points is called a "0-1-2-3 theory". Theories which extend down to points truly earn the adjective 'local'; other theories are only partially local. In this connection Segal [Se2] has observed that the existence of handlebody decompositions for manifolds means that in a theory with at least three "tiers", all of the data is determined in principle from balls and products of spheres. In another direction one can often build into a topological quantum field theory invariants for families of manifolds as well as invariants of a single manifold. String topology as well as the theory which encodes Gromov-Witten invariants both include invariants of families. One new development in this area are generators-and-relations structure theorems for TQFTs which are fully local - go down to points - and include families: see Theorem 3.12, Theorem 3.13, and Conjecture 3.14. In these statements the values of a TQFT are not necessarily Hilbert spaces and complex numbers. For these are really generators-and-relations results about bordism categories of manifolds, so the codomain can be very general. This flexibility in the codomain is necessary if one is to avoid semisimplicity when considering theories over rings which are not fields (see Remark 3.6).

In an $n$-dimensional theory the invariant of an $n$-manifold is a number and the invariant of an $(n-1)$-manifold is a set (the quantum Hilbert space). It is natural, then, that the invariant of an $(n-2)$-manifold be a category; the linear structure of quantum theory demands that it be a linear category. Further descent in dimension, obligatory for full locality, requires a concomitant ascent in category number. This is a familiar ladder one climbs - or descends, depending on your orientation - in 
topology. The simplest invariant of a space $S$ is the set $\pi_{0} S$ of path components. The next invariant is a category: the fundamental groupoid $\pi_{\leq 1} S$. At the next level we encounter a 2-category $\pi_{\leq 2} S$ and so on. To incorporate families of manifolds, one enriches to topological categories or some equivalent.

Chern-Simons theory as initially conceived from the path integral is a 2-3 theory. Reshetikhin and Turaev [RT, $[\mathrm{Tu}]$ give a rigorous mathematical construction when the gauge group is simple and simply connected; it utilizes a suitable category of representations of a related quantum group. Hence they construct a 1-2-3 theory; this category is attached to $S^{1}$. It has been a long-standing question to extend to a 0-1-2-3 theory: What does Chern-Simons attach to a point? In $\$ 4$ we give the answer for a finite gauge group (it was already contained in F2 ). There is an extension of these ideas which works when the gauge group is a torus group, and hopefully in more general cases as well.

In Chern-Simons theory the vector space attached to the torus $S^{1} \times S^{1}$ refines to a free abelian group which carries a ring structure, the so-called Verlinde ring. In a series of papers [FHT1, this ring was identified with a certain $K$-theory ring built directly from the gauge group; see Theorem 5.4. This opens the possibility of an a priori construction of quantum Chern-Simons theory using pure topology, specifically $K$-theory. There is an a priori construction of the 2 -dimensional reduction, as recounted at the end of $\$ 5$ and it is pure $K$-theory. It remains to be seen if this can be extended to an a priori 3 -dimensional construction, or at least if the input to the generators-and-relations Conjecture 3.14 can be given in terms of $K$-theory (as it is for finite groups and tori).

We begin in $\$ 1$ with a brief account of the differential geometry into which the classical Chern-Simons invariant fits; the actual invariant is constructed in the appendix. The mathematics which goes into the rigorous constructions of the quantum theory - quantum groups and the like - are quite different: only the path integral approach brings in the differential geometry directly. We emphasize this in 96 where we observe that an elementary consequence of the path integral (6.5), which involves classical invariants of flat connections, is not captured by the axiomatics and in fact remains unproven. This state of affairs returns us to a more general discussion about the interaction of mathematics with quantum field theory and string theory. The casual reader may wish to rejoin us at that point. In between we extract the formal structure of path integrals (\$2), present axiomatics for TQFT together with generators-and-relations theorems (\$3), discuss constructions of Chern-Simons as a 1-2-3 and 0-1-2-3 theory (\$4), and present the relationship to $K$-theory (\$5).

I had the good fortune to be a graduate student at Berkeley when MSRI opened its doors in 1982. From the beginning Chern nurtured an open and welcoming atmosphere. I quickly discovered that whereas faculty members at Evans Hall-as at any mathematics department - were occupied with teaching and administration as well as research, the visitors to MSRI were engaging with mathematics and (even young) mathematicians all day long. I learned early the world of difference between a mathematics department and a Mathematical Sciences Research Institute. No wonder so many institutes around the world emulate MSRI! Over its first 25 years, MSRI has grown tremendously in scope and outreach, all the while cultivating a creative environment for mathematics and mathematicians to flourish. I am very 
grateful for the time I have been able to spend at MSRI, and it is an honor to dedicate this paper to the continued good health of MSRI.

The circle of ideas surrounding Chern-Simons theory has been the topic of discussions with my collaborators Michael Hopkins and Constantin Teleman for at least eight years, and their influence can be felt throughout this paper as can the influence of earlier collaborators and my students. For the more recent topics, Jacob Lurie and David Ben-Zvi have joined the conversation. It is a pleasure to thank them all.

\section{The Classical Chern-Simons invariant}

We begin with the nineteenth century progenitors of the Chern-Weil and ChernSimons work. Let $\Sigma$ be a surface embedded in 3-dimensional Euclidean space. The curvature of $\Sigma$ is measured by a single function $K: \Sigma \rightarrow \mathbb{R}$. For example, the curvature of a sphere of radius $R$ is the constant function with value $1 / R^{2}$. The curvature was first investigated by Gauss in 1825. His famous theorema egregium [G. p. 105] proves that this Gauss curvature is intrinsic: it only depends on the induced metric on the surface, not on the embedding of the surface into space. The Riemannian metric determines a measure $d \mu_{\Sigma}$ on $\Sigma$. The Gauss-Bonnet theorem, apparently first given its global formulation by Walter van Dyck [Hi, p. 141], states that if $\Sigma$ is closed - compact with no boundary - then

$$
\int_{\Sigma} K d \mu_{\Sigma}=2 \pi \chi(\Sigma)
$$

where $\chi(\Sigma)$ is the Euler characteristic of $\Sigma$. This formula is the apogee of a first course in differential geometry. It and its many generalizations link local geometry and global topology.

Now suppose $\Sigma$ has a boundary $\partial \Sigma$ and is compact. Then there is an extra term which appears in (1.1), the total geodesic curvature of the boundary. Suppose $C \subset \Sigma$ is a closed curve equipped with an orientation of its normal bundle. The geodesic curvature $\kappa_{C}: C \rightarrow \mathbb{R}$ is a generalization of the curvature of a plane curve; it vanishes if $C$ is a geodesic. The metric induces a measure $d \mu_{C}$ on $C$ and

$$
\int_{C} \kappa_{C} d \mu_{C}
$$

is the total geodesic curvature of $C$. The generalized Gauss-Bonnet formula is

$$
\int_{\Sigma} K d \mu_{\Sigma}+\int_{\partial \Sigma} \kappa_{\partial \Sigma} d \mu_{\partial \Sigma}=2 \pi \chi(\Sigma) .
$$

The classical Chern-Simons invariant CSi] is a generalization of the total geodesic curvature (1.2). The version we need is defined on a compact oriented 3 -manifold $X$. Like the total geodesic curvature it is an extrinsic invariant, but now the extrinsic geometry is defined by a principal bundle with connection on $X$, not by an embedding into a Riemannian manifold. The differential geometry is quite pretty, and for the convenience of the reader we give a lightening review of connections and the general Chern-Simons construction in the appendix. For the exposition in the next section we use a simplified version of this classical invariant as our emphasis is on its quantization and ultimately the very different mathematics used to compute in the quantum theory. 


\section{Path integrals}

Let $G=S U(n)$ be the Lie group of unitary $n \times n$ matrices of determinant one for some $n \geq 2$. Its Lie algebra $\mathfrak{g}$ consists of $n \times n$ skew-Hermitian matrices of trace zero. Fix a closed oriented 3 -manifold $X$. A connection $A$ on the trivial $G$-bundle over $X$ is a skew-Hermitian matrix of 1 -forms with trace zero, i.e., $A \in \Omega^{1}(X ; \mathfrak{g})$. In this case the classical Chern-Simons invariant of $A$ is given by the explicit formula

$$
S(A)=\frac{1}{8 \pi^{2}} \int_{X} \operatorname{trace}\left(A \wedge d A+\frac{2}{3} A \wedge A \wedge A\right),
$$

where the wedge products are combined with matrix multiplication. The integrand in (2.1) is a 3-form, and the integral depends on the orientation of $X$. As mentioned earlier, this is a 3-dimensional analog of the 1-dimensional total geodesic curvature (1.2); there are generalizations to higher dimensions and all Lie groups, as explained in the appendix. Chern and Simons were particularly interested in the Levi-Civita connection on the tangent bundle of a Riemannian manifold $X$, in which case the Chern-Simons invariant is an obstruction to certain conformal immersions. They embarked on that study to derive combinatorial formulas for the first Pontrjagin number of a compact oriented 4-manifold, or perhaps with an eye toward the Poincaré conjecture. Regardless, the classical Chern-Simons invariant has found numerous applications in differential geometry, global analysis, topology, and theoretical physics.

Edward Witten W1 used the classical Chern-Simons invariant to derive not an invariant of Riemannian 3-manifolds, but rather a topological invariant of 3manifolds. The classical approach to remove the dependence of (2.1) on the connection $A$ is to treat $S(A)$ as defining a variational problem and to find its critical points. That is indeed interesting: the Euler-Lagrange equation asserts that the connection $A$ is flat. There is not in general a unique flat connection, so there is no particular critical value to choose as a topological invariant, though many interesting topological invariants may be formed from the space of flat connections. (They make an appearance in 6 , ) Witten's approach is quantum mechanical à la Feynman: he integrates out the variable $A$ to obtain a topological invariant. For each integer $k$, termed the level of the theory, set

$$
F_{k}(X) "=" \int_{\mathcal{F}_{X}} e^{i k S(A)} d A
$$

The integral takes place over a space of equivalence classes of connections. This is the quotient $\mathcal{F}_{X}$ of the infinite-dimensional linear space $\Omega^{1}(X ; \mathfrak{g})$ by a nonlinear action of the infinite-dimensional Lie group of maps $X \rightarrow G$. The action is given by the equation

$$
g \cdot A=g^{-1} A g+g^{-1} d g, \quad g: X \rightarrow G, \quad A \in \Omega^{1}(X ; \mathfrak{g}) .
$$

The result of the integral is a complex number $F_{k}(X)$.

We place quotation marks around the equality in (2.2) to indicate that the path integral is only a heuristic: the presumptive measure $d A$ in the notation would have to be constructed before making sense of (2.2). Shortly I will indicate approaches to rigorously defining topological invariants $F_{k}(X)$. Let me immediately point out a difficulty with the naive formula (2.2). If there really were a measure $d A$ which made that formula work, then we would conclude that $F_{k}(X)$ is a topological invariant of $X$ which depends only on the orientation of $X$. After all, the integrand-built 
from the classical Chern-Simons invariant (2.1) - only depends on the orientation. However, it turns out that $F_{k}(X)$ depends on an additional topological structure on $X$. It can variously be described as a 2 -framing [A1, a rigging [Se2], or a $p_{1^{-}}$ structure BHMV]. The dependence of the right-hand side of (2.2) on this structure signals the difficulties in defining the integral. Physicists approach the path integral through the process of regularization, which here can be accomplished by introducing a Riemannian metric on $X$. The regularization does not preserve all of the topological symmetry (orientation-preserving diffeomorphisms) of the classical action. One often says that the quantum theory has an anomaly. Interestingly, to obtain a topological invariant, albeit of a manifold with a $p_{1}$-structure, Witten introduces a "counterterm": the Chern-Simons invariant of the auxiliary Riemannian metric, precisely the invariant studied by Chern and Simons. While anomalies are well understood geometrically in many other contexts, this Chern-Simons anomaly remains somewhat of a mystery.

The implication of the previous may well be that one should construct a measure $d A$ on $\mathcal{F}_{X}$ which depends on a $p_{1}$-structure on $X$. As far as I know, this has not been done.

There is an important extension of the invariant (2.2) to 3-manifolds which contain an oriented link. (Again there is an anomaly: the normal bundle to the link must also be framed in the quantum theory.) Suppose that $\chi: G \rightarrow \mathbb{C}$ is a character of $G$. This means we represent $G$ linearly on a finite-dimensional vector space, and $\chi(g)$ is the trace of the matrix which represents the action of $g \in G$. Given a connection $A$ on $X$ and an oriented loop $C \subset X$, we define the holonomy of $A$ around $C$ by solving a first-order ordinary differential equation. The holonomy is only defined up to conjugacy, but since the character is invariant under conjugation we get a well-defined complex number $\chi_{C}(A)$. Given a link $L=\amalg C_{i}$ which is the finite disjoint union of oriented curves, the formal path integral definition is

$$
F_{k}(X, L) "=" \int_{\mathcal{F}_{X}} e^{i k S(A)} \prod \chi_{C_{i}}(A) d A .
$$

(One may choose a different character $\chi$ for each component of the link.) Witten shows how to compute the Jones polynomial invariant [Jo1] of a link in $S^{3}$ from the invariants $F_{k}(X, L)$ for $G=S U(2)$. Indeed, that was one of his motivations for this work. The invariants in (2.3) are a generalization of the Jones invariants to links in arbitrary 3-manifolds. They include as well the HOMFLYPT polynomial invariants FYHLMO, $\mathrm{PT}$.

In another direction the Chern-Simons theory is defined for any compact Lie group $G$; the level $k$ in (2.2) is replaced by a clas $\lambda \in H^{4}(B G ; \mathbb{Z})$, where $B G$ is the classifying space of the Lie group $G$. See the appendix for the classical theory in this generality. Note that $H^{4}(B S U(n) ; \mathbb{Z}) \cong \mathbb{Z}$ and there is a canonical generator.

How can we make mathematical sense out of (2.2)? One approach might be to attack directly the problem of constructing a measure $d A$. While this has worked well in other quantum field theories, the theory at hand is in the end topological and we might hope for a more topological approach. So instead we codify the structure inherent in (2.2) and seek to construct examples of that structure. In the remainder of this section we elucidate the bare formal properties of the path

\footnotetext{
${ }^{1}$ There is a nondegeneracy restriction. Namely, $H^{4}(B G ; \mathbb{R})$ is naturally isomorphic to the vector space of $G$-invariant symmetric bilinear forms on $\operatorname{Lie}(G)$, and we require that the image of $\lambda$ in this vector space be a nondegenerate form.
} 
integral. This leads to a set of axioms for a topological quantum field theory, which we discuss in $\S 3$. In $\$ 4$ we return to Chern-Simons theories and to their construction for various classes of Lie groups $G$.

To begin, it is important to extend to compact 3-manifolds $X$ with boundary. In that case we replace (2.2) by

$$
F_{k}(X)(\alpha) "=" \int_{\mathcal{F}_{X}(\alpha)} e^{i k S(A)} d A,
$$

where $\alpha$ is a connection on the boundary $\partial X$ and $\mathcal{F}_{X}(\alpha)$ is the space of gauge equivalence classes of connections on $X$ whose boundary value is $\alpha$. Thus $F_{k}(X)$ is a function on the space $\mathcal{F}_{\partial X}$ of gauge equivalence classes on $\partial X$.

We abstract the formal properties of (2.4) for a general path integral, not necessarily that in Chern-Simons theory. A quantum field theory lives in a fixed dimension $n$, which is the dimension of the "spacetimes" in the theory. For Chern-Simons theory $n=3$ and the theory is topological, but in general we should imagine our manifolds as having conformal structures or metrics, which we take to be Riemannian. Physicists call this "Euclidean field theory". Certainly time is not present in this positive definite signature - it is part of special relativity encoded in the Lorentz signature - so the use of "spacetime" in this Riemannian context should be understood by analogy with the Lorentzian case. Now for each spacetime $X$, which is an $n$-manifold with more structure, there is a space $\mathcal{F}_{X}$ of fields and a classical action 2

$$
S: \mathcal{F}_{X} \longrightarrow \mathbb{C} .
$$

The boundaries of spacetimes are closed $(n-1)$-manifolds $Y$, and on these we also imagine a space $\mathcal{F}_{Y}$ of fields (but no classical action). We want $Y$ to possess an infinitesimal normal bundle which is oriented: $Y$ comes equipped with an arrow of time. In the Riemannian case there is also a germ of a metric in the normal direction, but in a topological theory the normal orientation is enough. We consider a compact spacetime $X$ together with a decomposition of its boundary as a disjoint union $\partial X \cong Y_{0} \amalg Y_{1}$. The arrow of time on $Y_{0}$ points into $X$ and the arrow of time on $Y_{1}$ points out of $X$. Thus $Y_{0}$ is called the incoming part of the boundary and $Y_{1}$ the outgoing part. In topology we say $X$ is a bordism from $Y_{0}$ to $Y_{1}$ and write $X: Y_{0} \rightarrow Y_{1}$. These bordisms compose by gluing, which corresponds to the evolution of time. (See Figure 1,)

To a spacetime $X: Y_{0} \rightarrow Y_{1}$ the "semiclassical" field theory attaches a correspondence diagram

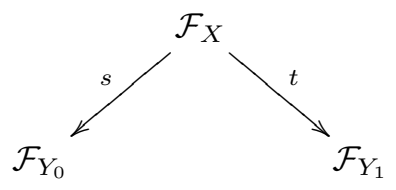

The source map $s$ and target map $t$ are simply restrictions of a field to the appropriate piece of the boundary. What is most important about the fields is locality.

\footnotetext{
${ }^{2}$ As we see from (2.2) it is only the exponentiated classical action which enters into the path integral; indeed, in Chern-Simons theory only that exponential is well defined. Also, in Euclidean field theory a minus sign usually appears in place of $i=\sqrt{-1}$ in 2.8 below, but we deliberately use notation which follows the example of Chern-Simons.
} 


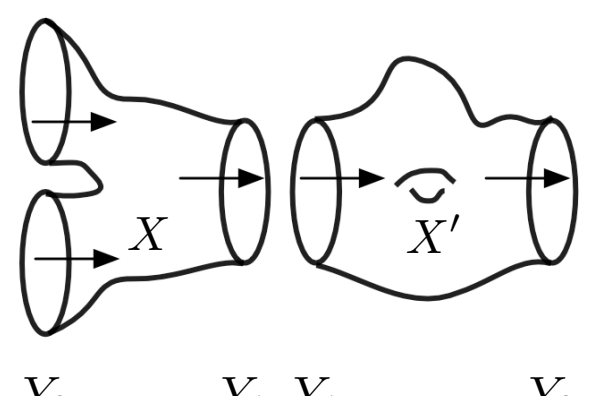

FiguRE 1. Gluing of bordisms

Suppose $X: Y_{0} \rightarrow Y_{1}$ and $X^{\prime}: Y_{1} \rightarrow Y_{2}$ are composable bordisms. Then in the diagram

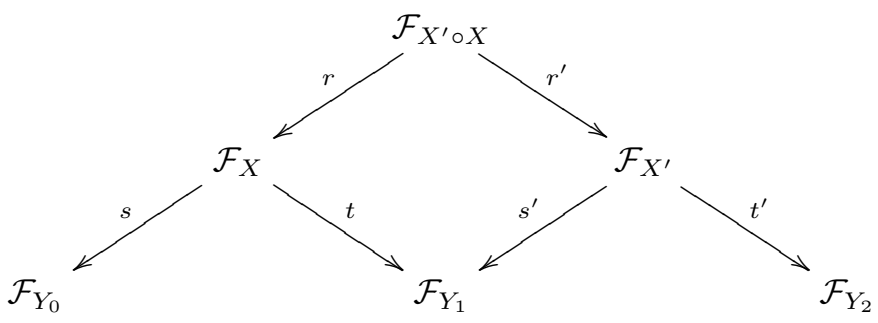

the space of fields $\mathcal{F}_{X^{\prime} \circ X}$ on the composition is the fiber product of the maps $t, s^{\prime}$. Loosely speaking, a field on the glued bordism is a pair of fields on the separate bordisms which agree along the common boundary. One should keep in mind that fields are really infinite-dimensional stacks. In other words, fields may have automorphisms, and these play an important role in this context. For example, in gauge theories such as Chern-Simons theory the gauge transformations act as morphisms of fields. Certainly automorphisms must be accounted for in the maps and fiber product in (2.5) and (2.6). The classical action is also assumed local in the sense that

$$
S_{X^{\prime} \circ X}(\Phi)=S_{X}(r(\Phi))+S_{X^{\prime}}\left(r^{\prime}(\Phi)\right) .
$$

It is worth noting that whereas the locality of the classical action is additive, that of the quantum theory is multiplicative. Crudely, quantization is a sort of exponentiation, as one can see from the integrand in (2.4).

Now we come to quantization in the geometric version given by the path integral. Assume there exist measures $\mu_{X}, \mu_{Y}$ on the spaces $\mathcal{F}_{X}, \mathcal{F}_{Y}$. Then define the Hilbert space $\mathcal{H}_{Y}=L^{2}\left(\mathcal{F}_{Y}, \mu_{Y}\right)$ and the linear map attached to a bordism $X: Y_{0} \rightarrow Y_{1}$ as the push-pull-with-kernel $e^{i S_{X}}$ :

$$
F_{X}=t_{*} \circ e^{i S_{X}} \circ s^{*}: \mathcal{H}_{Y_{0}} \longrightarrow \mathcal{H}_{Y_{1}} .
$$

The pushforward $t_{*}$ is integration. Thus if $f \in L^{2}\left(\mathcal{F}_{Y_{0}}, \mu_{Y_{0}}\right)$ and $g \in L^{2}\left(\mathcal{F}_{Y_{1}}, \mu_{Y_{1}}\right)$, then

$$
\left\langle\bar{g}, F_{X}(f)\right\rangle_{\mathcal{H}_{Y_{1}}}=\int_{\Phi \in \mathcal{F}_{X}} \overline{g(t(\Phi))} f(s(\Phi)) e^{i S_{X}(\Phi)} d \mu_{X}(\Phi) .
$$


If the boundary of $X$ is empty, then using the fact that $\mathcal{F}_{\emptyset}$ is a point, we see that (2.8) defines a complex number which is the path integral (2.2).

In short, the quantization (2.8) linearizes the correspondence diagram (2.5). This push-pull-with-kernel linearization of correspondence diagrams is ubiquitous in mathematics. The Fourier transform is the archetypal example. Let $S: \mathbb{R} \times \mathbb{R} \rightarrow \mathbb{R}$ be the duality pairing $S(x, \xi)=x \xi$ and $s, t: \mathbb{R} \times \mathbb{R} \rightarrow \mathbb{R}$ the two projections. Then the linearization of

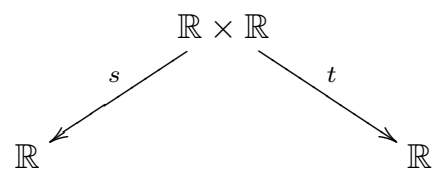

via (2.8) is the Fourier transform; the pushforward $t_{*}$ is integration with respect to suitably normalized Lebesgue measure.

The most important property of the path integral is multiplicativity under gluing. It asserts that for a composition $X^{\prime} \circ X$ of bordisms as in (2.6) we have

$$
F_{X^{\prime} \circ X}=F_{X^{\prime}} \circ F_{X}: \mathcal{H}_{Y_{0}} \longrightarrow \mathcal{H}_{Y_{2}} .
$$

This is a formal consequence of the fiber product in (2.6) and the additivity (2.7) if we assume an appropriate gluing law for the measures. As mentioned earlier, Chern-Simons can be defined for any compact Lie group $G$. If $G$ is a finite group, then the pushforward $t_{*}$ in (2.8) is a finite sum and the heuristics in this section can be carried out rigorously $\mathrm{FQ}$. Also, for a finite group there are no $p_{1}$-structures required and the quantum Chern-Simons theory is defined for oriented manifolds.

Finally, we remark that once quantum Chern-Simons theory is defined for 3manifolds with boundary, then invariants of links are included. Let $V$ be the Hilbert space attached to the standard torus $S^{1} \times S^{1}$. In Chern-Simons theory it is finite-dimensional, so we can fix a basis $e_{1}, e_{2}, \ldots, e_{N}$. Suppose $X$ is an oriented 3-manifold which contains an oriented link $L=\amalg C_{i}$ and that the normal bundle to each knot $C_{i}$ is framed. Let $X^{\prime}$ be the compact 3-manifold with boundary obtained from $X$ by removing a tubular neighborhood of $L$. The orientation and normal framing identifies each boundary component of $X^{\prime}$ with the standard torus. This identification is defined up to isotopy, and the topological invariance of ChernSimons theory implies that this is enough to define an isomorphism of the Hilbert space of $\partial X^{\prime}$ with $\otimes_{i} V$. View $\partial X^{\prime}$ as incoming. Then $F_{X^{\prime}}: \otimes_{i} V \rightarrow \mathbb{C}$. Suppose each component $C_{i}$ of $L$ is labeled by a basis vector $e_{i}$. Define

$$
F_{k}(X, L)=F_{k}\left(X^{\prime}\right)\left(\otimes_{i} e_{i}\right) .
$$

The constructions of Chern-Simons theory as a 1-2-3 theory produce a canonical basis of $V$ and assign a basis element to the characters $\chi$ which appear in (2.3).

\section{Axiomatization And 2-Dimensional theORIES}

Atiyah [A2 introduced a set of axioms for a topological quantum field theory (TQFT) which is patterned after Segal's axiomatic treatment Se2] of conformal field theory. Quinn Q gave a more elaborate treatment with many elementary examples. In recent years the concept of a TQFT has broadened and the definition has evolved. The most functorial modern definition of a topological quantum field theory (TQFT) makes evident both the analogy with homology theory and the 
formal properties of the path integral outlined in $\$ 2$. Recall that homology is a functor

$$
H:(T o p, \amalg) \longrightarrow(A b, \oplus)
$$

from the category of topological spaces and continuous maps to the category of abelian groups and homomorphisms. Both Top and Ab are symmetric monoidal categories: the tensor product on Top is disjoint union $\amalg$ and on $A b$ is direct sum $\oplus$. The functor $H$ is a symmetric monoidal functor: homology is additive under disjoint union. The codomain tensor category may be replaced by, for example, the category $V e c t_{\mathbb{F}}$ of vector spaces over a field $\mathbb{F}$ where the monoidal structure is direct sum of vector spaces. We remark that a full list of axioms for homology includes the Mayer-Vietoris property.

In a TQFT the category of topological spaces is replaced by a bordism category of manifolds of fixed dimension. As discussed in $₫ 2$ to compose bordisms we need an "arrow of time", which we term a collaring. Thus a collar of a closed manifold $Y$ is an embedding $Y \hookrightarrow \hat{Y}$ into a manifold $\hat{Y}$ which is diffeomorphic to $(-\epsilon, \epsilon) \times Y$ together with an orientation of the normal bundle of $Y \subset \hat{Y}$. If $Y$ is a collared manifold, then the opposite collared manifold $-Y$ is the same embedding $Y \subset \hat{Y}$ with the reversed orientation on the normal bundle. The boundary of a manifold $X$ has a collar [Hi, §4.6]; the arrow of time points out of $X$. Let $\operatorname{Bord}_{n}^{O}$ denote the category whose objects are compact collared $(n-1)$-manifolds $Y$. A morphism $Y_{0} \rightarrow Y_{1}$ is a compact $n$-manifold $X$ with a decomposition of its boundary $\partial X=$ $(\partial X)_{\text {in }} \amalg(\partial X)_{\text {out }}$ and collar-preserving diffeomorphisms $-Y_{0} \rightarrow(\partial X)_{\text {in }}$ and $Y_{1} \rightarrow$ $(\partial X)_{\text {out }}$. Bordisms $X, X^{\prime}$ define the same morphism if there exists a diffeomorphism $X \rightarrow X^{\prime}$ which commutes with the other data. Composition is gluing of bordisms. Precise definitions are given in GMWT, for example. The disjoint union $\amalg$ of manifolds endows $\operatorname{Bord}_{n}^{O}$ with a monoidal structure.

Definition 3.1. An $n$-dimensional topological quantum field theory $F$ is a symmetric monoidal functor

$$
F:\left(\operatorname{Bord}_{n}^{O}, \amalg\right) \longrightarrow(A b, \otimes) .
$$

Note that the tensor structure on abelian groups is tensor product: TQFT is multiplicative, whereas homology is additive. As with homology we can contemplate other codomains, for example replacing $(A b, \otimes)$ with $\left(V e c t_{\mathbb{F}}, \otimes\right)$ or $(R-\bmod , \otimes)$ for some commutative ring $R$. See Remark 3.6 below for a slightly more sophisticated replacement. One can go all the way and simply declare that the codomain is an arbitrary symmetric monoidal category, as in Theorem 3.12 below. The domain $\left(\operatorname{Bord}_{n}^{O}, \amalg\right)$ may be replaced by another bordism category: oriented bordism $\left(\right.$ Bord $\left.^{S O}, \amalg\right)$, spin bordism (Bord $\left.{ }^{S p i n}, \amalg\right)$, framed bordism (Bord $\left.{ }^{\text {fr }}, \amalg\right)$, etc.

The empty set is a manifold of any dimension; the empty manifold of dimension $n-1$ is a unit in $\left(\operatorname{Bord}_{n}^{O}, \amalg\right)$. It follows that $F(\emptyset)=\mathbb{Z}$ for any TQFT $F$. A closed oriented manifold $X$ of dimension $n$ is a morphism $X: \emptyset \rightarrow \emptyset$ from which $F(X) \in \mathbb{Z}$. An $n$-dimensional TQFT therefore assigns to closed manifolds algebraic objects as follows:

\begin{tabular}{cc}
\hline $\begin{array}{c}\text { closed manifold } \\
\text { of dim }\end{array}$ & $F(\cdot)$ \\
\hline$n$ & element of $\mathbb{Z}$ \\
$n-1$ & $\mathbb{Z}$-module \\
\hline
\end{tabular}


We consider now oriented TQFTs: the domain category is $\operatorname{Bord}_{n}^{S O}$. An object $Y$ is a closed oriented $(n-1)$-manifold with a collar $Y \hookrightarrow \hat{Y}$, and this induces an orientation on the $n$-manifold $\hat{Y}$. The opposite manifold $-Y$ has the opposite orientation and the opposite collar. The structure of a 1-dimensional oriented TQFT is quite simple. Let $F\left(\mathrm{pt}_{ \pm}\right)=A_{ \pm}$be the abelian groups assigned to a point with orientation. There is a unique closed oriented interval, up to diffeomorphism, but it defines four distinct morphisms in $\operatorname{Bord}_{1}^{S O}$ :

$$
\begin{aligned}
B= & F(\Im): A_{+} \otimes A_{-} \longrightarrow \mathbb{Z}, \\
B^{\vee}= & F(\mathcal{C}): \mathbb{Z} \longrightarrow A_{-} \otimes A_{+}, \\
& F(\hookrightarrow): A_{+} \longrightarrow A_{+}, \\
& F(\multimap): A_{-} \longrightarrow A_{-} .
\end{aligned}
$$

We read the pictures from left to right: the left boundary is incoming and the right boundary outgoing. It is easy to prove that the last two morphisms are idempotents. Assume they are identity maps; if necessary replace $A_{ \pm}$with the images of the idempotents. Then an easy gluing argument proves that

$$
\begin{aligned}
& A_{+} \stackrel{\text { id } \times B^{\vee}}{\longrightarrow} A_{+} \otimes A_{-} \otimes A_{+} \stackrel{B \times \mathrm{id}}{\longrightarrow} A_{+}, \\
& A_{-} \stackrel{B^{\vee} \times \mathrm{id}}{\longrightarrow} A_{-} \otimes A_{+} \otimes A_{-} \stackrel{\mathrm{id} \times B}{\longrightarrow} A_{-}
\end{aligned}
$$

are identity maps. It follows that (i) $A_{ \pm}$are free and finitely generated; (ii) $B$ determines an isomorphism $A_{-} \cong \operatorname{Hom}\left(A_{+}, \mathbb{Z}\right)$; and (iii) $F\left(S^{1}\right)=\operatorname{rank} A_{+}$. These arguments persist in an $n$-dimensional theory: take the Cartesian product with a fixed $(n-1)$-manifold $Y$.

Proposition 3.5. Let $F$ be a TQFT defined on $\operatorname{Bord}_{n}^{S O}$. Then for any closed oriented $(n-1)$-manifold $Y$, the abelian group $F(Y)$ is free and finitely generated, there is a duality between $F(Y)$ and $F(-Y)$, and $F\left(S^{1} \times Y\right)=\operatorname{rank} F(Y)$.

Remark 3.6. Proposition 3.5 already indicates the restrictive nature of Definition 3.1 the abelian groups $F(Y)$ are free. To construct a theory which includes torsion, we can replace the target tensor category $(A b, \otimes)$ with the tensor category $(\mathrm{dg}-A b, \otimes)$ of differential graded abelian groups; we take the differential to have degree 1 . The symmetry in $(\mathrm{dg}-A b, \otimes)$ uses the Koszul sign rule. Then there is a 1-dimensional theory with

$$
\begin{aligned}
& A_{+}: \cdots \longrightarrow 0 \longrightarrow \mathbb{Z} \stackrel{n}{\longrightarrow} \mathbb{Z} \longrightarrow 0 \longrightarrow 0 \longrightarrow \cdots, \\
& A_{-}: \cdots \longrightarrow 0 \longrightarrow 0 \longrightarrow \mathbb{Z} \stackrel{n}{\longrightarrow} \mathbb{Z} \longrightarrow 0 \longrightarrow \cdots,
\end{aligned}
$$

where the nonzero homogeneous groups in $A_{+}$are in degrees $-1,0$ and in $A_{-}$in degrees $0,+1$. Let $e_{-1}, e_{0}$ and $f_{0}, f_{1}$ be the obvious basis elements in (3.7). Set $B\left(e_{0}, f_{0}\right)=B\left(e_{-1}, f_{1}\right)=1$ and $B^{\vee}(1)=f_{1} \otimes e_{-1}+f_{0} \otimes e_{0}$. It is instructive to check (3.4) and compute $F\left(S^{1}\right)=0$. The complex $A_{+}$is a resolution of $\mathbb{Z} / n \mathbb{Z}$ : in this sense $F\left(\mathrm{pt}_{+}\right)$is torsion.

There is a richer, but still simple, structure for $n=2$. Recall that a Frobenius ring is a unital ring $A$ equipped with a homomorphism $\theta: A \rightarrow \mathbb{Z}$ which satisfies $\theta(x y)=\theta(y x)$ for all $x, y \in A$, and the pairing $x, y \mapsto \theta(x y)$ is nondegenerate. 
Theorem 3.8. A 2-dimensional TQFT $F$ determines a finitely generated commutative Frobenius ring $A=F\left(S^{1}\right)$. Conversely, a finitely generated commutative Frobenius ring $A$ determines a 2-dimensional TQFT $F$ with $F\left(S^{1}\right)=A$.

The statement of Theorem 3.8 can be found in many references, e.g., Dijkgraaf's thesis $\mathrm{D}$. A precise proof is given in $\mathrm{Ab}$; see also [MS, §A.1]. A stronger statement - an equivalence of categories of 2-dimensional TQFTs and finitely generated commutative Frobenius rings - is proved in $[\mathrm{K}]$. We exhibit this theorem as the paradigmatic generators-and-relations construction of a TQFT. The generators are the unit, trace, multiplication, and comultiplication, which give

$$
F(\bigcirc), \quad F(D), \quad F\left(\sum\right), \quad F(\Leftarrow),
$$

respectively. The commutativity and associativity relations assure that when any bordism $X$ is decomposed as a composition of these basic bordisms and $F(X)$ is defined as a composition of the generating data, then $F(X)$ is independent of the decomposition.

The notion of a TQFT, and the generators-and-relations Theorem 3.8, can be extended in various directions. We can, for example, ask for invariants of families of manifolds in addition to single manifolds. To see what this entails, suppose $F:\left(\operatorname{Bord}_{n}^{S O}, \amalg\right) \rightarrow\left(V e c t_{\mathbb{C}}, \otimes\right)$ is an $n$-dimensional TQFT. Let $y \rightarrow S$ be a fiber bundle with fiber a closed oriented $(n-1)$-manifold. Then the vector spaces $F\left(y_{s}\right)$ attached to fibers $y_{s}$ fit together into a flat vector bundle which we denote $F(y / S) \rightarrow S$. Local trivializations and the flat structure are derived from parallel transport: if $\gamma:[0,1] \rightarrow S$ is a smooth path from $s_{0}$ to $s_{1}$, then $\gamma^{-1} y: y_{s_{0}} \rightarrow y_{s_{1}}$ is a bordism and $F\left(\gamma^{-1} y\right): F\left(y_{s_{0}}\right) \rightarrow F\left(y_{s_{1}}\right)$ is defined to be parallel transport. Topological invariance shows that it is invariant under homotopies of $\gamma$. If $X \rightarrow S$ is a family of bordisms from $y_{0} \rightarrow S$ to $y_{1} \rightarrow S$, then applying $F$ we obtain

$$
F(X / S) \in H^{0}\left(S ; \operatorname{Hom}\left(F\left(y_{0} / S\right), F\left(y_{1}, S\right)\right)\right):
$$

the topological invariance implies that $F(X / S)$ is a flat section. It is natural, then, to extend $F$ to a $\mathbb{Z}$-graded functor $\tilde{F}$ which assigns a cohomology class

$$
\tilde{F}(X / S) \in H^{\bullet}\left(S ; \operatorname{Hom}\left(F\left(y_{0} / S\right), F\left(y_{1}, S\right)\right)\right)
$$

to a family $x \rightarrow S$. In addition to the functoriality under composition of morphisms, we require naturality under base change. This notion appears for $n=2$ in $[\mathrm{KM}$ where the invariants are extended to nodal (better: Deligne-Mumford stable) Riemann surfaces as well. Gromov-Witten invariants are an example of such a functor. There is a large literature on this subject: see $[\mathrm{T}$ for a recent result about the structure of 2-dimensional theories in families.

In another direction we can ask that an $n$-dimensional TQFT extend to manifolds of lower dimension or higher codimension. This idea dates from the early 1990s, when such theories were sometimes termed "extended TQFTs" [L, [F1]. They also go by the appellation "multi-tier theories" Se1. For $n=2$, a 3-tier theory is as far as we can go: it assigns invariants to points. We prefer to call this a "0-1-2 theory". Whatever the moniker, a 3-tier $n$-dimensional theory attaches a category to a manifold of codimension 2 (dimension $n-2)$. If we work over a ring $R$, then 
the table (3.2) is extended to the following:

\begin{tabular}{cc}
\hline $\begin{array}{c}\text { closed manifold } \\
\text { of dim }\end{array}$ & $F(\cdot)$ \\
\hline$n$ & element of $R$ \\
$n-1$ & $R$-module \\
$n-2$ & $R$-linear category \\
\hline
\end{tabular}

(The morphism sets in an $R$-linear category are $R$-modules and composition is an $R$-linear map.) The entire structure is 2-categorical: the domain is a 2-category whose objects are closed $(n-2)$-manifolds, 1-morphisms are $(n-1)$-manifolds, and 2 -morphisms are $n$-manifolds. Families of manifolds may also be incorporated using a topological version of a 2-category. One possibility is an infinity 2-category, which is a simplicial set with extra structure. An infinity 1-category, also called a weak Kan complex or quasicategory [BV], Joy, [Lu, may be used to encode an ordinary TQFT for families of manifolds. Other models, such as Segal's $\Gamma$-spaces, may be more convenient. The codomain can be an arbitrary symmetric monoidal infinity 1 - or infinity 2-category.

Remark 3.10. The discussion of ordinary 0-1 TQFTs surrounding (3.3) may be restated in these terms: a TQFT $F:\left(\operatorname{Bord}^{S O}, \amalg\right) \rightarrow \mathcal{C}$ with codomain a symmetric monoidal category $\mathcal{C}$ is determined up to isomorphism by a dualizable object $A \in \mathcal{C}$. Then $F\left(\mathrm{pt}_{+}\right)=A$ and the rest of the theory is determined by specifying the duality data. For $\mathcal{C}=(A b, \otimes)$ the dualizable objects are finitely generated free abelian groups.

Remark 3.11. One can also enrich homology theory to include categories analogously to chart (3.9). But there is a very important difference with quantum field theory. The categories which occur in this way in homology theory are groupoidsall morphisms are invertible. In quantum field theories the more general notion of duality replaces invertibility.

The structure of 0-1-2 theories, sometimes only partially defined, has recently been elucidated by Costello [C], Moore-Segal [MS], and others. Building on this work, Hopkins and Lurie prove a generators-and-relations theorem for 0-1-2 theories, in essence giving generators and relations for the infinity 2-category of 0 -, 1-, and 2-manifolds. (They also give an analogous result for 0-1 theories.) The simplest result to state is for framed manifolds and was conjectured by Baez-Dolan [BD]: the infinity 2-category of $0-, 1-$, and 2-manifolds with framing 3 is freely generated by a single generator, a framed point. The precise statement is in terms of functors to an arbitrary codomain.

Theorem 3.12 (Hopkins-Lurie). Let $\mathrm{C}$ be a symmetric monoidal infinity 2-category. Then the space of 0-1-2 theories of framed manifolds with values in $\mathcal{C}$ is homotopy equivalent to the space $\mathrm{C}^{\mathrm{fd}}$ of fully dualizable objects in $\mathrm{C}$.

The notion of a fully dualizable object in a symmetric monoidal $n$-category generalizes that of a dualizable object in a symmetric monoidal 1-category. The statement for a 0-1-2 theory is simpler than for a 1-2 theory (Theorem 3.8): there is now

\footnotetext{
${ }^{3}$ Precisely, the framing is a trivialization of the tangent bundle made 2-dimensional by adding a trivial bundle. For example, the framing of a point is a choice of basis of $\mathbb{R}^{2}$.
} 
a single generator and no relation. There is an explicit map which attaches to a field theory $F$ the fully dualizable object $F\left(\mathrm{pt}_{+}\right)$, and this is proved to be a homotopy equivalence. One powerful feature of the theorem is its "infinity aspect": it applies to families and so contains information about the diffeomorphism groups. The most important immediate application of this theorem is to string topology [CS; it implies that string topology produces homotopy invariants of manifolds. (Invariants from string topology which go beyond the homotopy type are thought to exist if one extends to nodal surfaces $\mathrm{Su}$.)

There is a generalization of Theorem 3.12 to other bordism categories. The orthogonal group $\mathrm{O}_{2}$ acts on the infinity 2-category of framed 0-, 1-, and 2-manifolds by rotating and reflecting the framing. Therefore, by Theorem $3.12, \mathrm{O}_{2}$ also acts on $\mathcal{e}^{\mathrm{fd}}$.

Theorem 3.13. Let $G \rightarrow \mathrm{O}_{2}$ be a homomorphism. Then the space of 0-1-2 theories of $G$-manifolds with values in $\mathcal{C}$ is homotopy equivalent to the space of homotopy $G$-fixed points in $\mathrm{C}^{\mathrm{fd}}$.

A 0-1-2 theory goes all the way down to points, so it is as local as possible. This explains why the structure of 0-1-2 theories is ultimately simpler than that of 1-2 theories. The principle that theories down to points are simpler inspires the program of Stolz-Teichner [ST, which aspires to construct a quantum field theory model of the generalized cohomology theory known as tmf [Ho, which is closely related to elliptic cohomology theories. These 3-tier theories also occur in two-dimensional conformal field theory: a category of "D-branes" is attached to a point in those theories.

Lurie has a detailed plan of proof for the conjectural generalization of Theorem 3.12 to higher dimensions.

Added in Proof. A forthcoming manuscript by Jacob Lurie gives precise definitions, examples, and an outline of the proof of Conjecture 3.14 now called the "cobordism hypothesis".

Conjecture 3.14 (Baez-Dolan-Lurie). Let $\mathrm{C}$ be a symmetric monoidal infinity ncategory. Then the space of $0-1 \cdots-n$ theories of framed manifolds with values in $\mathrm{C}$ is homotopy equivalent to the space $\mathrm{C}^{\mathrm{fd}}$ of fully dualizable objects in $\mathrm{C}$.

The obvious generalization of Theorem 3.13 is also conjectured to hold. The most local generators-and-relations constructions of 3-dimensional Chern-Simons theory will rely on Conjecture 3.14

\section{3-DIMENSIONAL TQFT}

We do not know a structure theorem for 2-3 theories à la Theorem 3.8 , Following the principle that a 3-tier theory is more local than a 2-tier theory, and hence simpler, there $i s$ a structure theorem for 1-2-3 theories. It is proved for theories defined 4 over $\mathbb{C}$ : the truncated $2-3$ theory maps into $\left(V e c t_{\mathbb{C}}, \otimes\right)$. An early version of this result is contained in an influential manuscript of Kevin Walker [Wa], which builds on earlier work of Moore and Seiberg [MSi]; there was also work of KazhdanReshetikhin along these lines, and probably work of others as well. A definitive version is in the book of Turaev [Tu], expanding on Reshetikhin-Turaev [RT].

\footnotetext{
${ }^{4}$ Work-in-progress by Davidovich, Hagge, and Wang aims to prove that any 1-2-3 theory over $\mathbb{C}$ may be defined over a number field.
} 
Theorem 4.1. A 1-2-3 theory $F$ determines a modular tensor category $\mathcal{C}=F\left(S^{1}\right)$. Conversely, a modular tensor category $\mathcal{C}$ determines a 1-2-3 theory $F$ with $F\left(S^{1}\right)=$ $\mathcal{C}$.

Loosely, a modular tensor category is the categorification of a commutative Frobenius algebra. More precisely, it is a braided monoidal category with duals and a ribbon structure. The category is required to be semisimple and there is a nondegeneracy condition as well. See [Tu] or [BK] for details. Theorem 4.1] is again a generators-and-relations construction: the monoidal structure tells $F($ ) the braiding tells $F$ applied to the diffeomorphism of $\Rightarrow$ which exchanges the two incoming boundary components by a half-turn, the duality tells $F$ applied to reflection on the circle, and the ribbon structure is related to the generator of $\pi_{1}\left(\right.$ Diff $\left.^{+} S^{1}\right) \cong \pi_{1}\left(S O_{2}\right) \cong \mathbb{Z}$. The statement of Theorem 4.1 is incomplete: we have not told on which bordism category these theories are defined. In fact, the 1-, 2-, and 3-manifolds in the domain of $F$ carry not only an orientation but also a $p_{1}$-structure. Together the orientation and $p_{1}$-structure are almost a framing, but we do not trivialize $w_{2}$ 居

The prime example and main object of interest for us is Chern-Simons theory, which was introduced heuristically in $\$ 2$ Recall that the data which defines the classical Chern-Simons invariant is a compact Lie group $G$ and a class $\lambda \in H^{4}(B G)$ termed the level. For $G$ a finite group the path integral construction of a 2-3 theory is rigorous [DW], FQ as the integral in this case is a finite sum. The path integral was extended to higher codimensions in [F2 to construct a 1-2-3 theory for $G$ finite. In fact, the discussion in that work extends all the way down to points, i.e., to a 0-1-2-3 theory, as we review presently. These are a priori constructions of the Chern-Simons TQFT for finite gauge groups. For continuous gauge groups the only known constructions are with generators and relations using Theorem 4.1. The modular tensor category $\mathcal{C}=\mathcal{C}(G, \lambda)$ should be the category of positive energy representations of the loop group at level $\lambda$ with the fusion product, but I do not believe any detailed construction along these lines has been carried out. The main theorem in FHT1 indicates that it should also be a categorification of the twisted equivariant $K$-theory of $G$ (with $G$ action by conjugation and the twist derived from $\lambda$ ), but this too has not been carried out. Rather, rigorous constructionsgoing back to Reshetikhin and Turaev [RT] - for $G$ simply connected and simple take as starting point the quantum group associated to the complexified Lie algebra of $G$ at a root of unity determined by $\lambda$. In particular, this gives a rigorous construction of the $S U(n)$-quantum invariants $F_{k}(X)$ introduced heuristically via the path integral in (2.4). There is a separate line of development based on operator algebras; see the paper of Jones Jo2 in this volume for an account. The theories for $G$ a torus are quite interesting and have been classified by Belov-Moore [BM]. The corresponding modular tensor categories are special - every simple object is invertible. The relation between these modular tensor categories and toral Chern-Simons theory is explored in Sti. The classical toral theories are specified by a finitely generated lattice with an even integer-valued bilinear form, whereas the quantum theories only remember a quadratic form on a finite abelian group extracted from the classical data. Thus the map from classical theories to quantum theories is many-to-one.

\footnotetext{
${ }^{5}$ There are generalizations of Chern-Simons theory defined on framed manifolds [J], and it will be interesting to have a structure theorem analogous to Theorem 4.1 for such 1-2-3 theories.
} 
Remark 4.2. A modular tensor category is semisimple. As we saw in Remark 3.6 we need to alter the codomain to escape semisimplicity, even in a 2-tier theory. The 3-dimensional Rozansky-Witten theory [RW], Ro], which takes as starting data a complex symplectic manifold, is not semisimple so does not fall under Theorem 4.1. Ongoing work of Kapustin-Rozansky-Saulina investigates the extension of Rozansky-Witten theory to a 0-1-2-3 theory.

A longstanding open question follows: Extend Chern-Simons theory down to points. In other words: Construct a 0-1-2-3 theory whose 1-2-3 truncation is ChernSimons theory for given $(G, \lambda)$. A glance at the charts (3.2) and (3.9) indicate the deep categorical waters ahead: a 4 -tier $n$-dimensional TQFT makes the following assignments:

\begin{tabular}{ccc}
\hline $\begin{array}{c}\text { closed manifold } \\
\text { of dim }\end{array}$ & $F(\cdot)$ & category number \\
\hline$n$ & element of $R$ & -1 \\
$n-1$ & $R$-module & 0 \\
$n-2$ & $R$-linear category & 1 \\
$n-3$ & $R$-linear 2-category & 2 \\
\hline
\end{tabular}

We attach a category number to each object in the chart: an $n$ category has category number $n$, a set has category number 0 , and an element in a set has category number -16 It is a feature of many parts of geometry over the past 25 years that the category number of objects and theorems has increased. Whereas theorems about equivalence classes - sets - used to be sufficient, new questions demand that automorphisms be accounted for: whence categories. This trend has affected-some would say infected - parts of quantum field theory as well. In our current context it appears that a 0-1-2-3 TQFT attaches a dualizable object in a symmetric monoidal 3 -category to an oriented point.

Fortunately, it may be possible to extend Chern-Simons down to points using only ordinary 1-categories. As motivation, one categorical level down we observe that a ring $R$ determines a 1-category, the category of $R$-modules. Similarly, a monoidal 1-category $\mathcal{R}$ determines a 2 -category of its modules. We can hope that in a given 0-1-2-3 theory $F$, such as Chern-Simons theory, the 2-category $F\left(\mathrm{pt}_{+}\right)$is the category of $\mathcal{R}$-modules for a monoidal 1 -category $\mathcal{R}$. We further speculate that if so, then $F\left(S^{1}\right)$ is the Drinfeld center $Z(\mathcal{R})$ of $\mathcal{R} 7$ The Drinfeld center of any monoidal category is braided, and in favorable circumstances $[\mathrm{Mu}]$ it is a modular tensor category. As evidence in favor of these hypotheses in the case of ChernSimons theory, we exhibit $\mathcal{R}$ in case the gauge group $G$ is finite and show that the modular tensor category $F_{(G, \lambda)}\left(S^{1}\right)$ is its Drinfeld center.

To begin we recall the precise definition of the center.

\footnotetext{
${ }^{6} \mathrm{I}$ once joked that every mathematician also has a category number, defined as the largest integer $n$ such that (s)he can think hard about $n$-categories for a half-hour without contracting a migraine. When I first said that my own category number was one, and in the intervening years it has remained steadfastly constant, whereas that of many around me has climbed precipitously, if not suspiciously.

${ }^{7}$ One can prove with reasonable hypotheses that $F\left(S^{1}\right)$ is the Hochschild homology of $\mathcal{R}$; the center is the Hochschild cohomology, and in favorable cases these may be identified.
} 
Definition 4.3. Let $\mathcal{R}$ be a monoidal category. Its Drinfeld center $Z(\mathcal{R})$ is the category whose objects are pairs $(X, \epsilon)$ consisting of an object $X$ in $\mathcal{R}$ and a natural transformation $\epsilon(-): X \otimes-\rightarrow-\otimes X$. The transformation $\epsilon$ is compatible with the monoidal structure in that for all objects $Y, Z$ in $\mathcal{R}$ we require

$$
\epsilon(Y \otimes Z)=\left(\operatorname{id}_{Y} \otimes \epsilon(Z)\right) \circ\left(\epsilon(Y) \otimes \operatorname{id}_{Z}\right) .
$$

This notion of center was introduced by Joyal-Street [JS]. A recent paper by Ben-Zvi, Francis, and Nadler [BFN] extends this notion of a center to a much broader context and contains discussions pertinent to this paper.

We explicate (ii). Suppose $G$ is a finite group and $\lambda \in H^{4}(B G)$ a level. There is an a priori "path integral" construction of the associated quantum Chern-Simons theory $F_{(G, \lambda)}$ in $[\mathrm{F} 2$ which starts with the classical Chern-Simons invariant for a finite group. The fields in this theory are principal $G$-bundles which, since $G$ is finite, are covering spaces with Galois group $G$. Over the circle the groupoid of principal $G$-bundles is equivalent to the groupoid $G / / G$ of $G$ acting on itself by conjugation. The level $\lambda$, through classical Chern-Simons theory, produces a central extension of this groupoid: for every pair of elements $x, y \in G$ a hermitian line $L_{x, y}$, for every triple of elements $x, y, z \in G$ an isomorphism

$$
L_{y x y^{-1}, z} \otimes L_{x, y} \longrightarrow L_{x, z y}
$$

and a consistency condition on the isomorphisms (4.4) for quartets of elements. Then $F_{(G, \lambda)}\left(S^{1}\right)$ is the category of $L$-twisted $G$-equivariant vector bundles over $G$. We remark that this is a concrete model for twisted $K$-theory, where the twisting is defined by $L$. (The relation of Chern-Simons theory to twisted $K$-theory is discussed in 95.) The monoidal structure is convolution, or pushforward under multiplication. We now exhibit this monoidal category as the center of a monoidal tensor category $\mathcal{R}_{(G, \lambda)}$.

First, view $H^{4}(B G)$ as $H^{2}\left(B G ; \mathbb{C P}^{\infty}\right)$ and so $\lambda$ as representing a central extension of $G$ by the abelian group-like (Picard) category of hermitian lines. A cocycle 8 which represents $\lambda$ is then a hermitian line $K_{x, y}$ for every pair of elements $x, y \in G$, cocycle isomorphisms

$$
K_{x, y} \otimes K_{x y, z} \longrightarrow K_{x, y z} \otimes K_{y, z}
$$

for triples of elements $x, y, z \in G$, and a consistency condition on these isomorphisms for quartets of elements in $G$. This is a categorified version of the usual cocycle for a central extension of a discrete group by the circle group. We normalize $K_{1, x}=K_{x, 1}=\mathbb{C}$ for all $x \in G$. Let $\mathcal{R}_{(G, \lambda)}$ be the category of complex vector bundles on $G$, or equivalently the category of $G$-graded complex vector spaces. If $W=\left\{W_{x}\right\}_{x \in G}$ and $W^{\prime}=\left\{W_{y}^{\prime}\right\}_{y \in G}$ are objects of $\mathcal{R}_{(G, \lambda)}$, set

$$
\left(W \otimes W^{\prime}\right)_{z}=\bigoplus_{x y=z} K_{x, y} \otimes W_{x} \otimes W_{y}^{\prime} .
$$

This monoidal structure on $\mathcal{R}_{(G, \lambda)}$ is a twisted convolution, and so $\mathcal{R}_{(G, \lambda)}$ is a twisted group algebra of $G$ with coefficients in the category of complex vector spaces. The dual $W^{*}$ of an object $W$ is defined as

$$
\left(W^{*}\right)_{x}=K_{x, x^{-1}}^{*} \otimes\left(W_{x^{-1}}\right)^{*} .
$$

\footnotetext{
${ }^{8}$ The construction, and so the theory, depends on the choice of cocycle up to noncanonical isomorphism. In the notation " $\lambda$ " should be understood to include the choice of cocycle.
} 
Then it is straightforward to check from Definition 4.3 that an object in the center $Z\left(\mathcal{R}_{(G, \lambda)}\right)$ of $\mathcal{R}_{(G, \lambda)}$ is a vector bundle $W \rightarrow G$ together with isomorphisms

$$
L_{x, y} \otimes W_{x} \longrightarrow W_{y x y^{-1}}
$$

where

$$
L_{x, y}:=K_{y x y^{-1}, y}^{*} \otimes K_{y, x} .
$$

Then (4.4) follows from (4.6), and (4.5) satisfies a consistency condition compatible with (4.4). This gives rise to a functor

$$
Z\left(\mathcal{R}_{(G, \lambda)}\right) \longrightarrow F_{(G, \lambda)}\left(S^{1}\right),
$$

and it is not hard to check that (4.7) is an equivalence of braided monoidal categories, in fact of modular tensor categories.

Remark 4.8. This construction appears in [F2] - see especially (9.16) thereinthough not in this language. The arguments in that reference make clear that $F_{(G, \lambda)}$ extends to a 0-1-2-3 theory with $F_{(G, \lambda)}\left(\mathrm{pt}_{+}\right)=\mathcal{R}_{(G, \lambda)}$-mod.

Remark 4.9. The modular tensor category $F_{(G, \lambda)}\left(S^{1}\right)$ is the category of representations of a Hopf algebra which is the Drinfeld double of a group algebra $\mathbb{C}[G, \lambda]$. The equivalence (4.7) is a categorified version: $F_{(G, \lambda)}\left(S^{1}\right)$ is the Drinfeld center of the twisted group algebra $\mathcal{R}_{(G, \lambda)}$ of $G$ with coefficients in $V e c t_{\mathbb{C}}$.

Remark 4.10. A variant of this construction works when $G$ is a torus group [FHLT]. Also, a different approach to Chern-Simons for a point using conformal nets is being developed by Bartels-Douglas-Henriques.

\section{Dimensional REDUCTiOn AND INTEGRALity}

A quantum field theory in $n$ dimensions gives rise to theories in a lower dimension through a process known as dimensional reduction. In classical field theory as practiced by physicists, fields in an $n$-dimensional theory are functions on $n$ dimensional Minkowski spacetime $M^{n}$. The entire theory is invariant under the Poincaré group $P^{n}$, which acts on $M^{n}$ by affine transformations. Dimensional reduction in this context [DF, $\S 2.11]$ is carried out by restricting the theory to fields which are invariant under a $k$-dimensional vector space $T$ of translations. Such fields drop to the quotient affine space $M^{n} / T$, which may be identified as $M^{n-k}$. The dimensionally reduced theory is invariant under the Poincaré group $P^{n-k}$. But there is an additional symmetry: the rotation group $S O(k)$ of the space $T$. In other words, the dimensional reduction of a theory has extra symmetry. In this section we explain a conjectural analog for topological quantum field theories.

Dimensional reduction may be carried out in quantum field theory as well. In the axiomatic framework this is easy, and for convenience we restrict to the dimensional reduction of a 3 -dimensional TQFT $F$ to a 2 -dimensional TQFT $F^{\prime}$. The definition is quite simple: for any manifold $M$ set

$$
F^{\prime}(M)=F\left(S^{1} \times M\right) \text {. }
$$

It is easy to check that this defines a 2-dimensional theory $F^{\prime}$. Suppose $F$ is a theory defined over the complex numbers; that is true for Chern-Simons theory, which is our main example.

Observation. For a closed 2-manifold $Y$ the complex number $F^{\prime}(Y)$ is an integer. 
This follows easily as $F^{\prime}(Y)=F\left(S^{1} \times Y\right)=\operatorname{dim} F(Y)$ is the dimension of a complex vector space, hence is a nonnegative integer 9 We record this in a chart:

\begin{tabular}{ccc}
\hline $\begin{array}{c}\text { closed manifold } \\
\text { of dim }\end{array}$ & $F(\cdot)$ & $F^{\prime}(\cdot)$ \\
\hline 3 & element of $\mathbb{C}$ & \\
2 & $\mathbb{C}$-vector space & element of $\mathbb{Z}$ \\
1 & $\mathbb{C}$-linear category & $\mathbb{Z}$-module \\
\hline
\end{tabular}

The last entry in the lower right-hand corner is speculative, as we do not know that the complex vector space $F\left(S^{1} \times S^{1}\right)$ can be refined to an abelian group. Nonetheless, we make the following

Conjecture 5.2. The dimensional reduction $F^{\prime}$ is defined over $\mathbb{Z}$.

In other words, there is a functor from a bordism category to $(A b, \otimes)$ whose composition with the functor $-\otimes_{\mathbb{Z}} \mathbb{C}:(A b, \otimes) \rightarrow\left(V e c t_{\mathbb{C}}, \otimes\right)$ is $F^{\prime}$.

To formulate Conjecture 5.2 precisely requires that we specify the precise nature of the linear category attached to a 1-manifold by the 1-2-3 theory $F$. We will not attempt that here, but report that with appropriate "smallness" hypotheses one can show that $F\left(S^{1} \times S^{1}\right)$ is the Hochschild homology of the linear category $F\left(S^{1}\right)$. Therefore, we seek an abelian group $A$ which refines the Hochschild homology in the sense that the latter is naturally $A \otimes_{\mathbb{Z}} \mathbb{C}$. This immediately brings to mind $K$-theory. Fortuitously, a similar integrality is required in noncommutative Hodge theory [KKP, §2.2.6]. There is a well-formulated plan, due to Bondal and Toën, to define the appropriate $K$-theory of appropriate dg-categories, based on work of Toën-Vaquie [TV] and the semitopological $K$-theory of Friedlander-Walker [FW] 10

Now that $K$-theory is on the table, we may be bolder and ask that $F^{\prime}$ map into the world of stable homotopy theory, which is a natural home for $K$-theory. In that world the integers are replaced by the sphere spectrum $S$, so at first we can replace "Z्Z" in the last column of (5.1) with " $S$ ". Modules over the sphere spectrum are spectra - just as modules over the integers are abelian groups - so we would have a TQFT with values in spectra. A more refined conjecture is that we can define $F^{\prime}$ to have values in $K$-modules, where " $K$ " denotes $K$-theory, an $E^{\infty}$ ring spectrum. In other words, we refine Conjecture 5.2 to

Conjecture 5.3. The dimensional reduction $F^{\prime}$ is defined over $K$.

We remark that spectra have appeared in the context of Floer homology CJS, [M], and most likely this fits into TQFT and Conjecture 5.3. Also, this refined conjecture is made in the noncommutative Hodge theory. Finally, one would naturally extend these speculations to propose a 0-1-2 theory $F^{\prime}$ over $K$. This seems quite mysterious, however, even in the explicit example of Chern-Simons for a finite group.

While these speculations are rather abstract, there is a theorem which provides some support. Looked at the other way, these speculations provide a context for the theorem, and indeed closely related considerations motivated it in the first

\footnotetext{
${ }^{9}$ We can consider theories in which the complex vector spaces are $\mathbb{Z} / 2 \mathbb{Z}$-graded. In that case we would not have nonnegativity of $F^{\prime}(Y)$.

${ }^{10}$ In fact, this $K$-theory group would refine the periodic cyclic homology, not the Hochschild homology, but these are closely related.
} 
place [F4]. Namely, consider the case of Chern-Simons theory $F$ for a compact Lie group $G$ and level $\lambda \in H^{4}(B G ; \mathbb{Z})$. Denote the reduction to a 2-dimensional theory by $F^{\prime}$. Then Conjecture 5.3 suggests that $F^{\prime}\left(S^{1}\right)$ is a $K$-theory group and can even be refined to a spectrum which is a $K$-module. Sticking for the moment to abelian groups, notice from Theorem 3.8 that $F^{\prime}\left(S^{1}\right)$ is a Frobenius ring. In this context physicists call this the Verlinde ring, which first appeared in rational conformal field theory $[\mathrm{V}]$. Let us denote it as $R(G, \lambda)$.

Theorem 5.4 (Freed-Hopkins-Teleman). There is a natural isomorphism

$$
\Phi: R(G, \lambda) \longrightarrow K_{G}^{\tau(\lambda)}(G) .
$$

The right-hand side is the twisted equivariant $K$-theory of the Lie group $G$, where $G$ acts on itself by conjugation. The twisting $\tau(\lambda)$ is transgressed from $\lambda$ and then shifted by a constant twisting 11 See [FHT1 for a detailed development and proof.

Remark 5.6. In Theorem 5.4 the left-hand side $R(G, \lambda)$ is defined to be the free abelian group generated by positive energy representations of the loop group of $G$ at a fixed level; the product is the fusion product. This fits the Chern-Simons story if we construct the modular tensor category $F\left(S^{1}\right)$ as the category of these representations. The theorem itself lies at the intersection of representation theory and algebraic topology.

Remark 5.7. While the statement of Theorem 5.4 was motivated by this physics, the explicit map $\Phi$ was not. (There is other, closely related, physics which could serve as motivation: the supersymmetric WZW model [Mi].) The explicit formula for $\Phi$ justifies its name: the Dirac family associated to a representation of the loop group. There is an analog for representations of the finite-dimensional group $G$. These Dirac families should find more applications in representation theory.

Remark 5.8. There is a refinement over $K$ in this case: one can make a $K$-module whose nontrivial homotopy group is $K_{G}^{\tau(\lambda)}(G)$.

Remark 5.9. While the 3-dimensional Chern-Simons theory $F$ is defined on a bordism category of oriented manifolds with $p_{1}$-structure, the 2-dimensional reduction $F^{\prime}$ factors to a theory of oriented manifolds: no $p_{1}$-structures.

The known rigorous constructions of the 3-dimensional Chern-Simons theory $F$ use generators and relations - for the 1-2-3 theory as encoded in a modular tensor category (Theorem 4.1) and for the 0-1-2-3 theory at least conjecturally in some cases (Conjecture 3.14, Remark 4.10). One attractive feature of the 2-dimensional reduction $F^{\prime}$ is that it has an a priori construction [FHT2] which follows the path integral heuristic discussed in 42 Recall that if $Y: S_{0} \rightarrow S_{1}$ is a 2-dimensional bordism, then the path integral linearizes the correspondence diagram

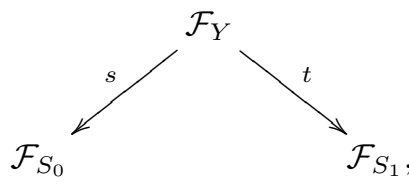

$$
t_{*} \circ e^{i S_{Y}} \circ s^{*}: L^{2}\left(\mathcal{F}_{S_{0}}\right) \longrightarrow L^{2}\left(\mathcal{F}_{S_{1}}\right) .
$$

Here $\mathcal{F}$ is the infinite-dimensional stack of $G$-connections. The definition of the $L^{2}$-spaces and the pushforward $t_{*}$ relies on measures which are consistent under

\footnotetext{
${ }^{11}$ This is the well-known shift $k \rightarrow k+n$ in the $S U(n)$-theories.
} 
gluing (and which do not usually exist, which is why this is a heuristic). Our $a$ priori construction replaces this path integral by a topological version:

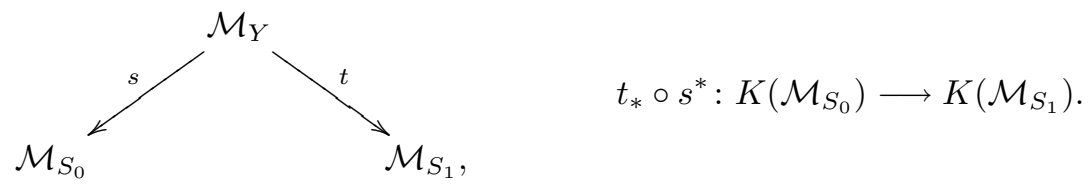

In this topological diagram the infinite-dimensional stack $\mathcal{F}$ of $G$-connections has been replaced by the finite-dimensional stack $\mathcal{M}$ of flat $G$-connections. The classical action $S$ vanishes. Also, the Hilbert spaces of $L^{2}$-functions are replaced by the abelian $K$-theory groups. The push-pull linearization is pure topology: the pullback $s^{*}$ is functorially defined and the pushforward $t_{*}$ depends on consistent $K$-theory orientations of the maps $t$. The consistency conditions refer to gluing of bordisms as expressed in diagram (2.6), but with the moduli stacks $\mathcal{M}$ of flat $G$-connections replacing the stacks $\mathcal{F}$ of all $G$-connections. It is important that flat $G$-connections are local in the sense that $\mathcal{M}_{X^{\prime} \circ X}$ is a fiber product, just as $\mathcal{F}_{X^{\prime} \circ X}$ is. This topological linearization of a correspondence diagram brings us full circle back to classical topology, though here we use $K$-theory in place of ordinary cohomology.

The moduli stack $\mathcal{M}_{S^{1}}$ of (flat) $G$-connections on the circle is equivalent to the global quotient $G / / G$ of $G$ acting on itself by conjugation. Therefore, $K\left(\mathcal{M}_{S^{1}}\right) \cong$ $K(G / / G) \cong K_{G}(G)$. A consistent orientation includes a twisting of this $K$-theory group, and in fact it is the group which occurs on the right-hand side of (5.5). This construction induces a Frobenius ring structure on this twisted equivariant $K$ theory group: the product is analogous to the Pontrjagin product on the homology of a topological group.

Remark 5.10. In FHT2 we prove that consistent orientations exist and are induced from a certain "universal orientation". The group of universal orientations maps to the group of levels, which is $H^{4}(B G ; \mathbb{Z})$, but in general that map is neither injective nor surjective. (It is a bijection for connected and simply connected groups such as $S U(n)$.) As the 2-dimensional reduction of Chern-Simons theory depends on a universal orientation, we can ask whether that is true of the whole 3-dimensional Chern-Simons theory, which is usually thought to depend only on the level.

Remark 5.11. We emphasize the analogy between measures and orientations. In our formal picture of the path integral, measures enable analytic integration. In this topological construction, orientations enable topological integration.

\section{Summary AND BRoAder PERspectives}

The current state of affairs for constructions of quantum Chern-Simons theories is as follows.

- There is a generators-and-relations construction of the 1-2-3 theory via modular tensor categories for many classes of compact Lie groups $G$. These include finite groups, tori, and simply connected groups, the latter via quantum groups or operator algebras (\$4).

- There are new generators-and-relations constructions - at this stage still conjectural - of the 0-1-2-3 theory for certain groups, including finite groups and tori (\$4). 
- There is an a priori construction of the 0-1-2-3 theory for a finite group (\$4).

- There is an a priori construction of the dimensionally reduced 1-2 theory for all compact Lie groups $G$ (\$5).

In many ways this represents a strong understanding of this particular 3-dimensional topological field theory. It is a small part of the successes mathematicians have achieved in understanding QFT-strings over the past 25 years. On the credit side too are the general structure theorems for topological field theories recounted in $₫ 3$ and 4 . All of this may be viewed as a triumph of the axiomatization of path integrals, which was motivated in $\$ 2$ and carried out in the succinct Definition 3.1. But we want to argue now that, in fact, this axiomatization falls far short of capturing all properties of the path integral. In particular, it fails for the Chern-Simons path integral, which is purely topological even at the classical level.

Remark 6.1. The beauty of the path integral heuristic is that it is an a priori definition of the topological invariants. Indeed, one of Witten's motivations in [W1] was to find a description of the Jones polynomial invariant of a link which manifestly exhibits 3-dimensional topological invariance. The bullet points above do not include an a priori rigorous construction of these invariants except for a finite gauge group, in which case the path integral heuristic can be expanded and made rigorous.

Another observation: the path integral takes as input the classical Chern-Simons invariant. But none of the mathematical constructions described in the bulleted list above uses this invariant save the a priori construction for a finite group.

To illustrate our lack of understanding, let us return to the Chern-Simons path integral (2.2) on a closed 3-manifold $X$ :

$$
F_{k}(X) “=" \int_{\mathcal{F}_{X}} e^{i k S(A)} d A .
$$

Now the usual expression for a path integral has integrand $e^{i S / \hbar}$, where $\hbar$ is Planck's constant. It is a constant of nature: $\hbar \sim 1.054572 \times 10^{-34} \mathrm{~m}^{2} \mathrm{~kg} / \mathrm{sec}$. But in this context we can consider the limit $\hbar \rightarrow 0$ in which quantum effects are suppressed, the so-called semiclassical limit. Mathematically, an oscillatory integral of the form

$$
F(\hbar)=\int e^{i S(x) / \hbar} d x
$$

has an asymptotic expansion as $\hbar \rightarrow 0$ which is derived by the method of stationary phase $[\mathrm{B}$. It is a sum over the critical points of $S$ if $S$ is a Morse function or an integral over the critical manifold if $S$ is Morse-Bott; in physics this sum is expressed in terms of Feynman diagrams and is the basis of perturbation theory and the many spectacular computations in quantum field theory. Comparing (6.2) and (6.3), we see the level $k$ plays the role of $1 / \hbar$. In other words, in this topological theory $\hbar$ takes on a countable set of values tending to zero. So the semiclassical limit is $k \rightarrow \infty$ and we expect an asymptotic expansion which is a sum over the critical points of $S$. Recall that the critical points of $S$ are the flat connections on $X$. Choose $G=S U(2)$ and suppose that $X$ is a closed oriented 3-manifold on which there is a finite set of equivalence classes $\mathcal{M}_{X}$ of flat $S U(2)$-connections. Let $\mathcal{M}_{X}^{0}$ denote the equivalence classes of irreducible flat $S U(2)$-connections, assumed 
nonempty. These contribute to the leading order asymptotics of $F_{k}(X)$ as $k \rightarrow \infty$ (see [W1], FG]).

Conjecture 6.4. The quantum Chern-Simons invariant satisfies

$$
F_{k}(X) \sim \frac{1}{2} e^{-3 \pi i / 4} \sum_{A \in \mathcal{M}_{X}^{0}} e^{2 \pi i S_{X}(A)(k+2)} e^{-2 \pi i I_{A} / 4} \sqrt{\tau_{X}(A)} .
$$

In (6.5) $S_{X}(A)$ is the classical Chern-Simons invariant (2.1), $I_{X}(A)$ is the AtiyahPatodi-Singer spectral flow [APS], and $\tau_{X}(A)$ is the Franz-Reidemeister torsion $[\mathbf{R}$, Fra.

Now to the point: The left-hand side is rigorously defined by the explicit construction of Chern-Simons theory using quantum groups. The right-hand side is a sum of classical invariants of flat connections. Thus, (6.5) is a well-formulated mathematical statement, a conjecture derived from the path integral. We offer the fact that this most basic consequence of the path integral is not proved as evidence that the axiomatics of $\$ 3$ do not capture all of its essential features. We will return to this line of thought presently.

Remark 6.6. The left-hand side of (6.5) is defined from the theory of quantum groups or loop groups. The computation of the exact quantum invariant, say for Seifert fibered manifolds, has as a crucial ingredient explicit formulas in that literature KaWa. On the other hand, the right-hand side involves three invariants of flat connections which are seemingly unrelated to quantum groups or loop groups. Conjecture 6.4 is typical of many from quantum field theory and string theory in that it relates parts of mathematics hitherto unconnected.

Remark 6.7. There are some special cases of Conjecture 6.4 which have been proved in the mathematics literature. The proofs go by examining the explicit formula for $F_{k}(X)$ and using techniques of classical analysis to derive the large $k$ asymptotics. For certain lens spaces this was carried out by Jeffrey Jef and Garoufalidis $\mathrm{Gar}$. Rozansky [Roz generalized to all Seifert fibered manifolds. Of course, most 3-manifolds are not Seifert fibered, and for these (6.5) remains a conjecture.

There is numerical evidence for Conjecture 6.4 for certain Seifert fibered manifold 12 [FG] and for some hyperbolic examples as well [Ha], [KSV]. Table 1 compares some explicit values of $F_{k}(X)$ and the approximation given by the asymptotic expansion in case $X$ is the Brieskorn homology sphere $X=\Sigma(2,3,17)$. The ratio of $F_{k}(X)$ to the predicted asymptotic value is close to one. We include this chart partly as an antidote to the abstractions we recounted from the formal properties of the path integral: the quantum Chern-Simons invariants are calculable! Indeed, these calculations were some of the first concrete evidence in a mathematical context that path integrals work as physicists claim. Yet this most basic statement about the path integral - the stationary phase asymptotic expansion - remains unproved in this topological example 13

There is much more to be said about the quantum Chern-Simons invariants in mathematics. For example, the deeper terms in the asymptotic expansion are also invariants of 3-manifolds (of "finite type"; see [Le for a survey) and links [B-N]. The latter were invented by Vassiliev [Va] in a different context. There have been

\footnotetext{
${ }^{12}$ This has been superseded by the analytical work in [Roz, as explained in Remark 6.7

${ }^{13}$ See $[\mathrm{CG}$ for some recent discussion.
} 
TABLE 1. Exact and asymptotic values for $X=\Sigma(2,3,17)$

\begin{tabular}{rrrc}
\hline$k$ & exact value $F_{k}(X)$ & \multicolumn{1}{c}{ asymptotic value } & \multicolumn{1}{c}{ ratio } \\
\hline 141 & $0.607899+0.102594 \mathrm{i}$ & $0.596099+0.151172 \mathrm{i}$ & $0.999182-0.081285 \mathrm{i}$ \\
142 & $-0.104966-0.151106 \mathrm{i}$ & $-0.094614-0.157913 \mathrm{i}$ & $0.997181-0.067244 \mathrm{i}$ \\
143 & $0.123614-0.139016 \mathrm{i}$ & $0.132261-0.128045 \mathrm{i}$ & $1.007707-0.075491 \mathrm{i}$ \\
144 & $-0.612014+0.038199 \mathrm{i}$ & $-0.614913-0.008261 \mathrm{i}$ & $0.994271-0.075479 \mathrm{i}$ \\
145 & $-0.291162-0.132171 \mathrm{i}$ & $-0.281928-0.153204 \mathrm{i}$ & $0.993986-0.071336 \mathrm{i}$ \\
146 & $-0.413944+0.674785 \mathrm{i}$ & $-0.465909+0.642185 \mathrm{i}$ & $0.994797-0.077144 \mathrm{i}$ \\
147 & $0.400490-0.286350 \mathrm{i}$ & $0.419276-0.254325 \mathrm{i}$ & $1.001116-0.075706 \mathrm{i}$ \\
148 & $-0.091879+0.669230 \mathrm{i}$ & $-0.143660+0.661309 \mathrm{i}$ & $0.995194-0.077257 \mathrm{i}$ \\
149 & $0.946786-0.263649 \mathrm{i}$ & $0.962119-0.191329 \mathrm{i}$ & $0.999048-0.075356 \mathrm{i}$ \\
150 & $-0.024553-0.058313 \mathrm{i}$ & $-0.021860-0.059113 \mathrm{i}$ & $1.002906-0.044484 \mathrm{i}$ \\
\hline
\end{tabular}

works too numerous to mention that study the mathematical structure of these invariants, including analytic properties of generating functions, number-theoretical properties, asymptotics of knot invariants, relations to hyperbolic geometry, etc. Probably the most innovative proposal involving Chern-Simons invariants is that they may be used in a model - both theoretical and practical - for quantum computation DFNSS. The Chern-Simons construction appears often in theoretical physics. One of the simplest uses is as a mass term in 3-dimensional abelian gauge theory. The Chern-Simons invariant of the Levi-Civita connection also appears in quantum theories of gravity [W2] in 3 dimensions. Higher-dimensional and higherdegree analogs of Chern-Simons appear in supergravity and string theory. The quantum Chern-Simons invariants are conjectured to have a relation to GromovWitten invariants [GV]. From the beginning they have been closely connected with 2-dimensional conformal field theory. And on and on.

Chern-Simons theory is one of many fronts over the last 25 years in a vigorous interaction between geometry and theories of quantum fields and quantum gravity. It has been a very fruitful period. The depth and variety of mathematics which has been applied to problems in physics is astounding. A new generation of theoretical physicists has a vast array mathematical tools at its fingertips, and these are being used to investigate many physical models. While the mathematical community is justifiably excited about new frontiers for applications of mathematics, such as biology, we should also keep in mind the impressive successes that contemporary applications of mathematics to fundamental physics enjoy.

What is particularly appealing for mathematicians in this interaction is the impact in the other direction: theoretical physics has opened up many new avenues of investigation in mathematics. Quantum field theory and string theory have made specific and concrete predictions that have motivated much mathematical work over this period. In part this is due to a temporary gulf between theory and physical experiments: in place of the traditional interplay between theorists and experimentalists has been an engagement with mathematics. Mathematicians have tested 
predictions of quantum field theory and string theory with independent mathematical techniques, in that way serving as experimentalists 14 But the ramifications for mathematics go much deeper. There are new connections between areas of mathematics and new lines of research in existing areas. It is a very exciting time.

However, it is hardly the moment to declare victory. What we find most exciting are the possibilities for the future, as we believe we have only just begun to absorb what this physics has to offer. To be more specific, one of the areas of success is topological aspects of quantum field theory, which includes the topological quantum field theories treated above. The axiomatics touched upon in $\$ 3$ encode a part of quantum field theory which applies beyond purely topological theories. Indeed, it is an old idea of 't Hooft ' $t$ ] that much can be learned about physical theories by exploring the topological information in the incarnations of a theory at different scales, and this idea has been valuable in many contexts. As we have seen, contemporary mathematical research in topological quantum field theory is yielding new structure theorems and interesting applications. Another exciting current area of mathematics-physics interaction relates the geometric Langlands program to 4-dimensional gauge theories [KW]. Lessons learned from 3-tier TQFTs, as represented in (3.9), have had a large impact here 15 Another broad area of success over the past 25 years has been in conformal field theory, particularly in two dimensions. There is a similar geometric axiomatization $[\mathrm{Se} 2$ as well as more algebraic approaches [FBZ, [BeDr] of which the three cited references are only the tip of the iceberg. Representation theory - most recently geometric parts of representation theory - has been one part of mathematics intimately connected with conformal field theory. Also, mirror symmetry in 2-dimensional conformal field theory and string theory [HKKPTVVZ] has had many consequences for algebraic geometry.

The common feature shared by topological and conformal theories is the absence of scale. Conformal invariance is precisely the statement that a theory looks the same at all scales. And topology is usually studied without introducing scale in the first place. Yet scale is one of the most basic concepts in all of physics, in particular in quantum field theory. Even if one begins with a theory which is conformally invariant at the classical level - and many important examples, such as Yang-Mills theory in 4-dimensions and $\sigma$-models in 2 dimensions, are - the process of regularization alluded to in $₫ 2$ introduces a scale into the theory. The dependence of the quantum theory on scale, encoded in the renormalization group [Wi], $\mathrm{Po}$, is a basic part of quantum field theory which guides every practicing physicist in the field. There is a body of mathematical work on quantum field theory (see [GJ] for one account) which focuses on foundational analytic aspects which include scale, but the newer investigations into topological and conformal features have been largely disjoint. The asymptotic expression (6.5) belongs to the part of quantum field theory which depends on scale: it is derived W1 through a geometric version

\footnotetext{
${ }^{14} \mathrm{As}$ this is being written, the LHC in Geneva is about to be turned on. There is a possibility that new data from that large machine will turn practitioners of modern mathematical physics back to a more traditional connection with experiment.

${ }^{15}$ In this $n=4$-dimensional topological field theory there is a category assigned to each Riemann surface. That category is argued to be one, or at least closely related to one, which enters the geometric Langlands program. There is a basic symmetry in 4-dimensional gauge theory, $S$-duality, which relates two different theories, and on a Riemann surface this is meant to induce the conjectured geometric Langlands correspondence between two different categories attached to the surface.
} 
of regularization together with standard manipulations of an integral. Its unrelieved conjectural status is only one of many signals that we need to turn more attention to the scale-dependent aspects of quantum field theory. I emphasize that this is important even in theories which are scale-independent. In particular, new ideas are needed to integrate the scale-dependent and scale-independent aspects.

We illustrate with an episode from 4-dimensional topology and field theory. In 1988, Witten W3 introduced a topological twisting of a supersymmetric gauge theory which encodes the Donaldson invariants Do of 4-manifolds. The underlying quantum field theory is not topological, but certain correlation functions in a twisted version on oriented Riemannian 4-manifolds compute Donaldson invariants. This new context for these invariants of smooth manifolds bore fruit six years later when Seiberg and Witten [SW] described the long-distance physics of the underlying quantum field theory. Their work is very much a part of scale-dependent physics: the long-distance approximation is a field theory with different fields than the fundamental short-distance theory. Seiberg and Witten use not only standard perturbation theory and renormalization group ideas, but also the supersymmetry which severely constrains the form of the long-distance physics. As the Donaldson invariants are topological, so independent of scale, they have an expression in both the long-distance and short-distance theories. In the short-distance theory one gets out Donaldson's definition, which is Witten's original work, but the long-distance approximation gives a new expression in terms of new equations - what are now known as the Seiberg-Witten equations. Geometers immediately ran with these new equations, which are simpler than the instanton equations used by Donaldson, and for many geometric purposes are all one needs. But it is important to realize that they are part of a scale-dependent story in quantum field theory. There has been considerable effort to prove the conjectured equivalence between the two sets of 4-manifold invariants, and very recently it was finally proved by Feehan and Leness $[\mathrm{FL}$ ] for 4-manifolds of simple type. (All known 4-manifolds are of simple type.) Here again we see that the discovery of scale-independent mathematics rests on a deep understanding of scale-dependence.

The recent solution of the Poincaré conjecture by Perelman $\mathrm{P}$ offers some insight. The statement of the theorem - a simply connected 3-manifold is homeomorphic to the 3-sphere - is very much in the realm of scale-invariant mathematics: no scale enters at all. Hamilton's Ricci flow $[\mathrm{H}]$ introduces scale on a smooth 3-manifold in the form of a Riemannian metric. That metric may exhibit irregularities at arbitrary small distances. The Ricci flow smooths out the small-scale fluctuations in the metric and, as time evolves, shifts focus to larger and larger scales. Topological consequences, such as Poincaré, are deduced in the infinite time limit when one approaches scale-independence. Of course, this is a poetic rendition of very intricate mathematics, especially as the flow may be interrupted by singularities. Still, it serves as a prototype for scale-dependence in quantum field theories, and for good reason: Ricci flow is the renormalization group flow for a particular quantum field theory, the 2-dimensional $\sigma$-model [Fr].

The past 25 years have seen much interaction between physics and algebraic, topological, and geometrical ideas. The addition of analytic ideas relevant to the study of scale will enrich this area in the years ahead. 


\section{Appendix: The Chern-Simons-Weil theory of COnneCtions}

Let $G$ be a Lie group with finitely many components. Let $\mathfrak{g}$ be its Lie algebra and $\pi: P \rightarrow M$ a principal $G$-bundle. Each element $g \in G$ acts on $P$ by a diffeomorphism $R_{g}: P \rightarrow P$. Let $\theta \in \Omega_{G}^{1}(\mathfrak{g})$ be the left-invariant Maurer-Cartan form; it transfers to any right $G$-torsor 16 Denote by $i_{m}: P_{m} \rightarrow P$ the inclusion of the fiber at $m \in M$.

Definition A.1. A connection on $P$ is a 1 -form $\Theta \in \Omega_{P}^{1}(\mathfrak{g})$ which satisfies

$$
R_{g}^{*} \Theta=\operatorname{Ad}_{g^{-1}} \Theta, \quad i_{m}^{*} \Theta=\theta
$$

for all $m \in M$.

The curvature of $\Theta$ is the $\mathfrak{g}$-valued 2 -form

$$
\Omega=d \Theta+\frac{1}{2}[\Theta \wedge \Theta]
$$

It satisfies the linear equations

$$
R_{g}^{*} \Omega=\operatorname{Ad}_{g^{-1}} \Omega, \quad i_{M}^{*} \Omega=0
$$

so lives in the linear space $\Omega_{M}^{2}\left(\mathfrak{g}_{P}\right)$ of 2 -forms on $M$ with values in the adjoint bundle of Lie algebras associated to $P$. Differentiating (A.3), we obtain the Bianchi identity $d \Omega+[\Theta \wedge \Omega]=0$. To work out these equations, the reader will use the Jacobi identity $[[\Theta \wedge \Theta] \wedge \Theta]=0$ and the structure equation $d \theta+\frac{1}{2}[\theta \wedge \theta]=0$.

The Chern-Weil construction is as follows. Fix

$$
\langle\rangle: \mathfrak{g}^{\otimes p} \longrightarrow \mathbb{R}
$$

which is $G$-invariant and symmetric.

Proposition A.5. $\omega(\Theta)=\langle\Omega \wedge \cdots \wedge \Omega\rangle$ is a closed $2 p$-form on $M$.

The proof is immediate: differentiate $\omega(\Theta)$ and use Bianchi and the $\mathfrak{g}$-invariance.

Example A.6. Let $\Sigma$ be an oriented Riemannian 2-manifold, $\pi: P \rightarrow \Sigma$ its $S_{2^{-}}$ bundle of oriented orthonormal frames, and $\Theta$ the Levi-Civita connection. Then $\langle\Omega\rangle=K \operatorname{vol}_{\Sigma}$, where vol is the Riemannian volume 2 -form. In this case $p=1$, and we define the linear map (A.4) to identify the Lie algebra of $\mathrm{SO}_{2}$ with $\mathbb{R}$. (We need not assume $M$ is oriented: the Chern-Weil construction for the $\mathrm{O}_{2}$-bundle of frames produces the density $K d \mu_{\Sigma}$ which appears in (1.1).)

The de Rham theorem tells that $\omega(\Theta)$ determines a cohomology class on $M$. It follows from (A.8) below that this class is independent of the connection, so it is a topological invariant of the principal bundle $\pi: P \rightarrow M$. More important to us here is the geometry which follows from the fact that the pullback $\pi^{*} \omega(\Theta)$ is exact. The construction of Chern and Simons CSi which we now recount produces a canonical antiderivative A.9), following their philosophy that "the manner in which a closed form which is zero in cohomology actually becomes exact contains geometric information" (quoted in [DGMS] 17

\footnotetext{
${ }^{16}$ A torsor for $G$ is a manifold on which $G$ acts simply transitively. The fibers of a principal bundle are right $G$-torsors.

${ }^{17}$ This is analogous to the rise of category number in geometry: the manner in which two sets are isomorphic contains geometric information.
} 
As (A.2) are affine equations, the space of solutions $\mathcal{A}_{P} \subset \Omega_{P}^{1}(\mathfrak{g})$ is also affine. It is the space of all connections on $P$. There is a universal connection $\Theta_{P}$ on $\mathcal{A}_{P} \times P \rightarrow \mathcal{A}_{P} \times M$ characterized by

$$
\left.\Theta_{P}\right|_{\{\Theta\} \times P}=\Theta,\left.\quad \Theta_{P}\right|_{\mathcal{A}_{P} \times\{p\}}=0, \quad \Theta \in \mathcal{A}_{P}, p \in P .
$$

Let $\Omega_{P}$ denote its curvature and $\omega\left(\Theta_{P}\right)$ its Chern-Weil form. Now any two connections $\Theta_{0}, \Theta_{1} \in \mathcal{A}_{P}$ are the endpoints of a line segment $\Delta^{1} \rightarrow \mathcal{A}_{P}$, an affine map with domain the standard 1-simplex. Define the Chern-Simons form

$$
\alpha\left(\Theta_{0}, \Theta_{1}\right)=\int_{\Delta^{1}} \omega\left(\Theta_{P}\right) \in \Omega_{M}^{2 p-1} .
$$

Stokes' formula implies

$$
d \alpha\left(\Theta_{0}, \Theta_{1}\right)=\omega\left(\Theta_{1}\right)-\omega\left(\Theta_{0}\right) .
$$

The affine structure of $\mathcal{A}_{P}$ allows us to continue to higher simplices: three connections determine a 2 -simplex and, by integration, a $(2 p-2)$-form whose differential relates the three Chern-Simons forms on the boundary, etc.

Our interest is to define a geometric invariant of a single connection. For this we work with the pullback bundle $\pi^{*} P \rightarrow P$. It has a canonical trivialization $\Delta: P \rightarrow \pi^{*} P$ : under the identification of $\pi^{*} P$ with the fiber product $P \times{ }_{M} P$, the section $\Delta$ is the diagonal map. This section defines a trivial connection $\Theta_{\Delta} \in \mathcal{A}_{\pi^{*} P}$ characterized by $\Delta^{*} \Theta_{\Delta}=0$. Pullback of connections defines an affine embedding $\pi^{*}: \mathcal{A}_{P} \hookrightarrow \mathcal{A}_{\pi^{*} P}$. For $\Theta \in \mathcal{A}_{P}$ define the Chern-Simons form

$$
\alpha(\Theta)=\alpha\left(\Theta_{\Delta}, \pi^{*} \Theta\right) \in \Omega_{P}^{2 p-1} .
$$

Then $\alpha(\Theta)$ is a differential form on $P$. From (A.8) we deduce

$$
d \alpha(\Theta)=\pi^{*} \omega(\Theta) \in \Omega_{P}^{2 p} .
$$

The construction is functorial. For example, applied to the unique connection on the principal bundle $G \rightarrow \mathrm{pt}$, we obtain a closed form $\alpha(\theta) \in \Omega_{G}^{2 p-1}$. The functoriality, together with (A.2), implies the second of the equations

$$
R_{g}^{*} \alpha(\Theta)=\alpha(\Theta), \quad i_{m}^{*} \alpha(\Theta)=\alpha(\theta) .
$$

Both (A.7) and (A.9) are termed "Chern-Simons forms"; the former lives on the base $M$ and depends on two connections, the latter lives on the total space $P$ and depends on a single connection.

Example A.10. In the situation of Example A.6, let $C \subset \Sigma$ be a smooth oriented curve. There is a canonical framing of $\Sigma$ along $C$ - the oriented tangent vector is the first basis vector - and so a canonical lift of $C$ to the frame bundle $P$. The Chern-Simons 1-form $\alpha(\Theta)$ then pulls down to a 1-form on $C$ which is $\kappa_{C} \operatorname{vol}_{C}$; compare (1.2).

The Chern-Simons invariant generalizes the total geodesic curvature (1.2). Suppose $\Theta$ is a connection on a principal $G$-bundle $\pi: P \rightarrow M$ with $M$ a closed oriented $(2 p-1)$-manifold. Let \langle\rangle be an invariant polynomial as in (A.4) and assume it is normalized so that the form $\alpha(\theta) \in \Omega_{G}^{2 p-1}$ has integral periods. Assume first that 
$\pi: P \rightarrow M$ is trivializable. Then if $s: M \rightarrow P$ is any section of $\pi$, the Chern-Simons invariant

$$
S(\Theta)=\int_{M} s^{*} \alpha(\Theta) \quad(\bmod 1)
$$

is independent of $s$. Note that the invariant $S(\Theta)$ lives in $\mathbb{R} / \mathbb{Z}$.

For a general (nontrivializable) bundle $\pi: P \rightarrow M$, we need a more intricate construction [F3, [DW]. Let $E G \rightarrow B G$ be a universal bundle built from smooth (Hilbert) manifolds, $\Theta^{\text {univ }}$ a connection on $E G$, and $\gamma: P \rightarrow E G$ a $G$-equivariant (classifying) map with quotient $\bar{\gamma}: M \rightarrow B G$. Since the odd homology of $B G$ is torsion, for some positive integer $N$ there is a smooth $2 p$-chain $W$ in $B G$ with $\partial W=$ $N \cdot \bar{\gamma}([M])$. Then $\left[\frac{1}{N} W\right] \in H_{2 p}(B G ; \mathbb{R} / \mathbb{Z})$. The Chern-Simons invariant depends on a choice of cohomology class $\lambda \in H^{2 p}(B G ; \mathbb{Z})$ whose image in $H^{2 p}(B G ; \mathbb{R})$ is represented by the universal Chern-Weil form $\omega\left(\Theta^{\text {univ }}\right)$. The integrality assumption on \langle\rangle guarantees the existence of $\lambda$. For a connection $\Theta$ on $P$ set

$$
S(\Theta)=\frac{1}{N} \int_{W} \omega\left(\Theta^{\text {univ }}\right)+\int_{M} \alpha\left(\gamma^{*} \Theta^{\text {univ }}, \Theta\right)+\lambda\left(\left[\frac{1}{N} W\right]\right) \in \mathbb{R} / \mathbb{Z} .
$$

One can check that the right-hand side is independent of the choices of $\gamma, W$ and defines a smooth function $S: \mathcal{A}_{P} \rightarrow \mathbb{R} / \mathbb{Z}$.

\section{ABout The AUThor}

Dan Freed is Professor of Mathematics at the University of Texas in Austin. His work in geometry and topology has close connections with quantum field theory and string theory.

\section{REFERENCES}

[Ab] L. Abrams, Two-dimensional topological quantum field theories and Frobenius algebras, J. Knot Theory Ramifications 5 (1996), no. 5, 569-587. MR1414088 (97j:81292)

[APS] M. F. Atiyah, V. K. Patodi, and I. M. Singer, Spectral asymmetry and Riemannian geometry. III, Math. Proc. Cambridge Philos. Soc. 79 (1976), no. 1, 71-99. MR.0397799(53:1655c)

[A1] M. F. Atiyah, On framings of 3-manifolds, Topology 29 (1990), no. 1, 1-7. MR 1046621 (91g:57025)

[A2] Topological quantum field theories, Inst. Hautes Études Sci. Publ. Math. No. 68 (1988), no. 68, 175-186 (1989). MR1001453 (90e:57059)

[BD] J. C. Baez, J. Dolan, Higher-dimensional algebra and topological quantum field theory, J. Math. Phys. 36 (1995), no. 11, 6073-6105, arXiv:q-alg/950300. MR.1355899 (97f:18003)

[BK] B. Bakalov, A. Kirillov, Jr., Lectures on tensor categories and modular functors, University Lecture Series, vol. 21, American Mathematical Society, Providence, RI, 2001. MR:1797619 (2002d:18003)

[B-N] D. Bar-Natan, Perturbative Chern-Simons theory, J. Knot Theory Ramifications 4 (1995), no. 4, 503-547. MR:1361082 (97c:58157)

[BeDr] A. Beilinson, V. Drinfeld, Chiral algebras, American Mathematical Society Colloquium Publications, vol. 51, American Mathematical Society, Providence, RI, 2004. MR 2058353 (2005d:17007)

[BM] D. Belov, G. Moore, Classification of abelian spin Chern-Simons theories, arXiv:hep-th/0505235.

[BFN] D. Ben-Zvi, J. Francis, D. Nadler, Integral transforms and Drinfeld centers in derived algebraic geometry, arXiv:0805.0157. 
[BHMV] C. Blanchet, N. Habegger, G. Masbaum, and P. Vogel, Topological quantum field theories derived from the Kauffman bracket, Topology 34 (1995), no. 4, 883-927. MR 1362791 (96i:57015)

[BV] J. M. Boardman, R. M. Vogt, Homotopy invariant algebraic structures on topological spaces, Lecture Notes in Mathematics, Vol. 347, Springer-Verlag, Berlin, 1973. MR0420609(54:8623a)

[B] R. Bott, Critical point theory in mathematics and in mathematical physics, Turkish J. Math. 21 (1997), no. 1, 9-40. MR1456156 (98k:58037)

[CS] M. Chas, D. Sullivan, String topology, arXiv:math/9911159, to appear in Annals of Math.

[Ch] S.-S. Chern, A simple intrinsic proof of the Gauss-Bonnet formula for closed Riemannian manifolds, Ann. of Math. (2) 45 (1944), 747-752. MR.0011027 (6:106a)

[CJS] R. L. Cohen, J. D. S. Jones, G. B. Segal, Floer's infinite-dimensional Morse theory and homotopy theory, The Floer Memorial Volume, Progr. Math., vol. 133, Birkhäuser, Basel, 1995, pp. 297-325. MR1362832 (96i:55012)

[C] K. Costello, Topological conformal field theories and Calabi-Yau categories, Adv. Math. 210 (2007), no. 1, 165-214, arXiv:math/0412149. MR2298823 (2008f:14071)

[CG] O. Costin, S. Garoufalidis, Resurgence of the Kontsevich-Zagier power series, arXiv: math/0609619.

[CSi] S.-S. Chern, J. Simons, Characteristic forms and geometric invariants, Ann. of Math. (2) 99 (1974), 48-69. MR0353327(50:5811)

[DFNSS] S. Das Sarma, M. Freedman, C. Nayak, S. H. Simon, A. Stern, Non-abelian anyons and topological quantum computation, arXiv:0707.1889.

[DF] P. Deligne, D. S. Freed, Classical field theory, Quantum fields and strings: a course for mathematicians, Vol. 1, 2 (Princeton, NJ, 1996/1997), Amer. Math. Soc., Providence, RI, 1999, pp. 137-225. MR.1701599(2000i:53109)

[DGMS] P. Deligne, P. Griffiths, J. Morgan, D. Sullivan, Real homotopy theory of Kähler manifolds, Invent. Math. 29 (1975), no. 3, 245-274. MR0382702 (52:3584)

$[\mathrm{D}]$ R. Dijkgraaf, A geometrical approach to two-dimensional conformal field theory, Ph.D. thesis.

[DW] R. Dijkgraaf, E. Witten, Topological gauge theories and group cohomology, Comm. Math. Phys. 129 (1990), no. 2, 393-429. MR.1048699 (91g:81133)

[Do] S. K. Donaldson, Polynomial invariants for smooth four-manifolds, Topology 29 (1990), no. 3, 257-315. MR1066174 (92a:57035)

[FL] P. M. N. Feehan, T. G. Leness, Witten's conjecture for four-manifolds of simple type, arXiv:math/0609530.

[Fe] R. P. Feynman, Space-time approach to non-relativistic quantum mechanics, Rev. Modern Physics 20 (1948), 367-387. MR0026940 (10:224b)

[Fra] W. Franz, Über die Torsion einer Überdeckung, J. Reine Angew. Math. 173 (1935), 245-254.

[FBZ] E. Frenkel, D. Ben-Zvi, Vertex algebras and algebraic curves, second ed., Mathematical Surveys and Monographs, vol. 88, American Mathematical Society, Providence, RI, 2004. MR 2082709 (2005d:17035)

[F1] D. S. Freed, Extended structures in topological quantum field theory, Quantum Topology, Ser. Knots \& Everything, vol. 3, World Sci. Publ., River Edge, NJ, 1993, pp. 162-173, arXiv:hep-th/9306045. MR.1273572 (95e:57039)

[F2] Higher algebraic structures and quantization, Comm. Math. Phys. 159 (1994), no. 2, 343-398, arXiv:hep-th/9212115. MR.1256993(95c:58034)

[F3] Leterminants, torsion, and strings, Comm. Math. Phys. 107 (1986), no. 3, 483-513. MR866202 (88b:58130)

[F4] The Verlinde algebra is twisted equivariant K-theory, Turkish J. Math. 25 (2001), no. 1, 159-167, arXiv:math/0101038. MR1829086(2002h:19007)

[FG] D. S. Freed, R. E. Gompf, Computer calculation of Witten's 3-manifold invariant, Comm. Math. Phys. 141 (1991), no. 1, 79-117. MR1133261 (93d:57027)

[FHT1] D. S. Freed, M. J. Hopkins, C. Teleman, Loop groups and twisted K-theory I, II, III, arXiv:0711.1906, arXiv:math/0511232, arXiv:math/0312155. 
[FHT2] Consistent orientation of moduli spaces, The many facets of geometry: a tribute to Nigel Hitchin, eds. J.P. Bourguignon, O. Garcia-Prada, S. Salamon, Oxford University Press, to appear, arXiv:0711.1909.

[FHLT] D. S. Freed, M. J. Hopkins, J. Lurie, C. Teleman, in preparation.

[FQ] D. S. Freed, F. Quinn, Chern-Simons theory with finite gauge group, Comm. Math. Phys. 156 (1993), no. 3, 435-472, arXiv: hep-th/911100. MR1240583(94k:58023)

[FYHLMO] P. Freyd, D. Yetter, J. Hoste, W. B. R. Lickorish, K. Millett, A. Ocneanu, A new polynomial invariant of knots and links, Bull. Amer. Math. Soc. (N.S.) 12 (1985), no. 2, 239-246. MR776477 (86e:57007)

[Fr] D. H. Friedan, Nonlinear models in $2+\epsilon$ dimensions, Annals Phys. 163 (1985), 318-419. MR811072 (87f:81130)

[FW] E. Friedlander, M. Walker, Semi-topological $K$-theory, Handbook of $K$-theory, Springer-Verlag, Berlin, 2005, pp. 877-924. MR2181835 (2006k:19008)

[GMWT] S. Galatius, I. Madsen, U. Tillmann, M. Weiss, The homotopy type of the cobordism category, arXiv:math/0605249.

[Gar] S. Garoufalidis, Relations among 3-manifold invariants, University of Chicago preprint.

[G] K. F. Gauss, (Translation by J. C. Morehead), General Investigations of Curved Surfaces, Princeton University Library, 1902, available at http://books . google.com/books?id=a1wTJR3kHwUC\&dq.

[GJ] J. Glimm, A. Jaffe, Quantum physics, second ed., Springer-Verlag, New York, 1987, A functional integral point of view. MR887102 (89k:81001)

[GV] R. Gopakumar, C. Vafa, Topological gravity as large $N$ topological gauge theory, Adv. Theor. Math. Phys. 2 (1998), no. 2, 413-442. MR1633024 (2000c:81254)

[H] R. S. Hamilton, Three-manifolds with positive Ricci curvature, J. Differential Geom. 17 (1982), no. 2, 255-306. MR664497 (84a:53050)

[Ha] R. J. Harrington, Witten's SU(2) invariant for a family of hyperbolic 3-manifolds, University of Texas Ph.D. thesis.

[Hi] M. W. Hirsch, Differential topology, Graduate Texts in Mathematics, vol. 33, Springer-Verlag, New York, 1994, Corrected reprint of the 1976 original. MR.1336822 (96c:57001)

[HKKPtVVZ] K. Hori, S. Katz, A. Klemm, R. Pandharipande, R. Thomas, C. Vafa, R. Vakil, E. Zaslow, Mirror symmetry, Clay Mathematics Monographs, vol. 1, American Mathematical Society, Providence, RI, 2003, With a preface by Vafa. MR2003030 (2004g:14042)

[Ho] M. J. Hopkins, Algebraic topology and modular forms, Proceedings of the International Congress of Mathematicians, Vol. I (Beijing, 2002) (Beijing), Higher Ed. Press, 2002, arXiv:math/0212397, pp. 291-317. MR1989190 (2004g:11032)

[Jef] L. C. Jeffrey, Chern-Simons-Witten invariants of lens spaces and torus bundles, and the semiclassical approximation, Comm. Math. Phys. 147 (1992), no. 3, 563604. MR.1175494 (93f:57042)

[J] J. A. Jenquin, Spin Chern-Simons and spin TQFTs, arXiv:math/0605239.

[Jo1] V. F. R. Jones, A polynomial invariant for knots via von Neumann algebras, Bull. Amer. Math. Soc. (N.S.) 12 (1985), no. 1, 103-111. MR766964(86e:57006)

[Jo2] $\quad$ On the origin and development of subfactors and quantum topology, Bull. Amer. Math. Soc. 46 (2009), no. 2, xx-xx.

[Joy] A. Joyal, Quasi-categories and Kan complexes, J. Pure Appl. Algebra 175 (2002), no. 1-3, 207-222, Special volume celebrating the 70th birthday of Professor Max Kelly. MR1935979 (2003h:55026)

[JS] A. Joyal, R. Street, Tortile Yang-Baxter operators in tensor categories, J. Pure Appl. Algebra 71 (1991), no. 1, 43-51. MR1107651 (92e:18006)

[KaWa $\quad$ V. G. Kac, M. Wakimoto, Modular and conformal invariance constraints in representation theory of affine algebras, Adv. in Math. 70 (1988), no. 2, 156-236. MR.954660 (89h:17036)

[KW] A. Kapustin, E. Witten, Electric-magnetic duality and the geometric Langlands program, Commun. Number Theory Phys. 1 (2007), no. 1, 1-236, arXiv:hep-th/0604151. MR2306566 (2008g:14018) 
[KSV] M. Karowski, R. Schrader, E. Vogt, Invariants of three-manifolds, unitary representations of the mapping class group, and numerical calculations, Experiment. Math. 6 (1997), no. 4, 317-352. MR1606924 (99g:57020)

[KKP] L. Katzarkov, M. Kontsevich, T. Pantev, Hodge theoretic aspects of mirror symmetry, arXiv:0806.0107v1.

[KM] M. Kontsevich, Yu. Manin, Gromov-Witten classes, quantum cohomology, and enumerative geometry, Comm. Math. Phys. 164 (1994), no. 3, 525-562, arXiv:hep-th/9402147. MR 1291244 (95i:14049)

[K] J. Kock, Frobenius algebras and 2D topological quantum field theories, London Mathematical Society Student Texts, vol. 59, Cambridge University Press, Cambridge, 2004. MR2037238 (2005a:57028)

[L] R. J. Lawrence, Triangulations, categories and extended topological field theories, Quantum Topology, Ser. Knots \& Everything, vol. 3, World Sci. Publ., River Edge, NJ, 1993, pp. 191-208. MR1273575 (95e:57040)

[Le] T. T. Q. Le, Finite type invariants of 3-manifolds, arXiv:math/0507145v1.

[Lu] J. Lurie, Higher Topos Theory, available at http://www-math.mit.edu/ lurie/.

[Ma] George W. Mackey, Unitary group representations in physics, probability, and number theory, second ed., Advanced Book Classics, Addison-Wesley Publishing Company Advanced Book Program, Redwood City, CA, 1989. MR:1043174 (90m:22002)

[M] C. Manolescu, Seiberg-Witten-Floer stable homotopy type of three-manifolds with $b_{1}=0$, Geom. Topol. 7 (2003), 889-932 (electronic), arXiv:math/0104024. MR2026550 (2005b:57060)

[Mi] J. Mickelsson, Gerbes, (twisted) K-theory, and the supersymmetric WZW model, Infinite dimensional groups and manifolds, IRMA Lect. Math. Theor. Phys., vol. 5, de Gruyter, Berlin, 2004, pp. 93-107, arXiv:hep-th/0206139. MR2104355 (2006c:58037)

[MS] G. W. Moore, G. B. Segal, D-branes and K-theory in 2D topological field theory, arXiv: hep-th/0609042.

[MSi] G. Moore, N. Seiberg, Polynomial equations for rational conformal field theories, Phys. Lett. B 212 (1988), no. 4, 451-460. MR962600 (89m:81155)

$[\mathrm{Mu}] \quad$ M. Müger, From subfactors to categories and topology II: The quantum double of tensor categories and subfactors, J. Pure Appl. Algebra 180 (2003), 159-219. MR.1966525 (2004f:18014)

[P] G. Perelman, The entropy formula for the Ricci flow and its geometric applications, arXiv:math/0211159.

[Po] J. Polchinski, Renormalization and effective Lagrangians, Nuclear Phys. B 231 (1984), 269-295.

[PT] J. H. Przytycki, P. Traczyk, Invariants of links of Conway type, Kobe J. Math. 4 (1988), no. 2, 115-139. MR.945888 (89h:57006)

[Q] F. Quinn, Lectures on axiomatic topological quantum field theory, Geometry and quantum field theory (Park City, UT, 1991), IAS/Park City Math. Ser., vol. 1, Amer. Math. Soc., Providence, RI, 1995, pp. 323-453. MR.1338394 (96e:57021)

[R] K. Reidemeister, Homotopieringe und Linsenrüme, Abh. Math. Sem. Univ. Hamburg 11 (1935), 102-109.

[RT] N. Reshetikhin, V. G. Turaev, Invariants of 3-manifolds via link polynomials and quantum groups, Invent. Math. 103 (1991), no. 3, 547-597. MR1091619 (92b:57024)

[Ro] J. Roberts, Rozansky-Witten theory, Topology and Geometry of Manifolds (Athens, GA, 2001), Proc. Sympos. Pure Math., vol. 71, Amer. Math. Soc., Providence, RI, 2003, pp. 1-17, arXiv:math/0112209. MR2024627 (2004m:57026)

[Roz] L. Rozansky, A large $k$ asymptotics of Witten's invariant of Seifert manifolds, Commun. Math. Phys. 171 (1995), 279-322, arXiv:hep-th/9303099. MR1344728 (96g:57036)

[RW] L. Rozansky, E. Witten, Hyper-Kähler geometry and invariants of threemanifolds, Selecta Math. (N.S.) 3 (1997), no. 3, 401-458, arXiv:hep-th/9612216. MR1481135 (98m:57041) 
[Se1] G. B. Segal, The locality of holomorphic bundles, and the locality of quantum field theory, The many facets of geometry: a tribute to Nigel Hitchin, eds. J.P. Bourguignon, O. Garcia-Prada, S. Salamon, Oxford University Press, to appear.

[Se2] The definition of conformal field theory, Topology, Geometry and Quantum Field Theory, London Math. Soc. Lecture Note Ser., vol. 308, Cambridge Univ. Press, Cambridge, 2004, pp. 421-577. MR2079383 (2005h:81334)

[SW] N. Seiberg, E. Witten, Electric-magnetic duality, monopole condensation, and confinement in $N=2$ supersymmetric Yang-Mills theory, Nuclear Phys. B 426 (1994), no. 1, 19-52. MR1293681 (95m:81202a)

[Sti] S. D. Stirling, Abelian Chern-Simons theory with toral gauge group, modular tensor categories, and group categories, arXiv:0807.2857.

[ST] S. Stolz, P. Teichner, What is an elliptic object?, Topology, Geometry and Quantum Field Theory, London Math. Soc. Lecture Note Ser., vol. 308, Cambridge Univ. Press, Cambridge, 2004, pp. 247-343. MR2079378 (2005m:58048)

[Su] D. Sullivan, String Topology: Background and Present, arXiv:0710.4141.

['t] G. 't Hooft, Naturalness chiral symmetry and spontaneous chiral symmetry breaking, Recent Developments in Gauge Theories (G't Hooft et al., ed.), Plenum Press, New York, 1980.

[T] C. Teleman, The structure of 2D semi-simple field theories, arXiv:0712.0160.

[TV] B. Toën, M. Vaquie, Moduli of objects in dg-categories, arXiv:math/0503269, to appear in Annales de l'ENS.

[Tu] V. G. Turaev, Quantum invariants of knots and 3-manifolds, de Gruyter Studies in Mathematics, vol. 18, Walter de Gruyter \& Co., Berlin, 1994. MR1292673 (95k:57014)

[Va] V. A. Vassiliev, Cohomology of knot spaces, Theory of Singularities and Its Applications, Adv. Soviet Math., vol. 1, Amer. Math. Soc., Providence, RI, 1990, pp. 23-69. MR.1089670 (92a:57016)

[V] E. Verlinde, Fusion rules and modular transformations in $2 D$ conformal field theory, Nuclear Phys. B 300 (1988), no. 3, 360-376. MR954762 (89h:81238)

[Wa] K. Walker, On Witten's 3-manifold invariants, available at http://canyon23. net/math/.

[Wi] K. G. Wilson, The renormalization group: critical phenomena and the Kondo problem, Rev. Modern Phys. 47 (1975), no. 4, 773-840. MR0438986 (55:11887)

[W1] E. Witten, Quantum field theory and the Jones polynomial, Comm. Math. Phys. 121 (1989), no. 3, 351-399. MR990772 (90h:57009)

[W2] $\quad 2+1$-dimensional gravity as an exactly soluble system, Nuclear Phys. B 311 (1988/89), no. 1, 46-78. MR974271 (90a:83041)

[W3] $\quad$ Topological quantum field theory, Comm. Math. Phys. 117 (1988), no. 3, 353-386. MR953828 (89m:57037)

Department of Mathematics, University of Texas, 1 University Station C1200, AusTin, TEXAs 78712-0257

E-mail address: dafr@math.utexas.edu 